\title{
Delay Feedback Control for Switching Diffusion Systems Based on Discrete Time Observations
}

\author{
Xiaoyue Li*and Xuerong Mao ${ }^{\dagger}$ and Denis S. Mukama ${ }^{\ddagger}$ and Chenggui Yuan ${ }^{\S}$
}

September 9, 2021

\begin{abstract}
Since response lags are required by most of physical systems and play a key role in the feedback control, the aim of this paper is to design delay feedback control functions based on the discrete-time observations of the system states and the Markovian states in order for the controlled switching diffusion system (SDS) to be exponentially stable in $p$ th moment and probability one as well as stable in $H_{\infty}$. The main methods are the strong ergodicity theory of Markov chains and asymptotic analysis of stochastic functional differential equations (SFDEs). For the sake of saving time and costs the feedback control based on discrete-time observations is used to stabilize the switching diffusion systems. The designed control principles are implementable to stablize quasi-linear and highly nonlinear SDSs. For quasi-linear SDSs the criteria are sharp that under the control with high strength the controlled SDSs will be stable (bounded) while under the weaker control they will be unstable (unbounded) in mean square. The sample and moment Lyapunov exponents are estimated which have close relationship with the time delays.
\end{abstract}

Key words. Brownian motion - Markov chain-Stochastic functional differential equations Exponential stability·Moment boundedness·Lyapunov functional

AMS subject classifications. $\quad 60 \mathrm{H} 10 \cdot 93 \mathrm{D} 15 \cdot 60 \mathrm{~J} 10$

\section{Introduction}

Switching diffusion systems (SDSs) modulated by Markov chains involving continuous dynamics and discrete events provide more realistic models to describe the systems in many branches of science and industry which experience abrupt changes in their structures and parameters. Because of the wide range of applications, dynamical properties of SDSs have been investigated extensively (see, e.g., $[2,15,26]$ and the references therein). It is

\footnotetext{
*School of Mathematics and Statistics, Northeast Normal University, Changchun, Jilin, 130024, China. Research the Fundamental Research Funds for the Central Universities.

${ }^{\dagger}$ Department of Mathematics and Statistics, University of Strathclyde, Glasgow G1 1XH, U.K.

${ }^{\ddagger}$ School of Mathematics and Statistics, Northeast Normal University, Changchun, Jilin, 130024, China.

$\S$ Department of Mathematics, Swansea University, Bay Campus, SA1 8EN, UK.
} 
due to the Markovian switching that the dynamics of SDSs may be drastically different from that of the systems without switching. For example, several counterexamples given in $[17,18]$ reveal that the recurrence or transience properties are opposite from their subsystems' without switching. For more properties such as the strong Feller, recurrence and stability please refer to [20], [25] and the references therein.

One of the important issues in the study of SDSs is the automatic control, with consequent emphasis being placed on the their stabilization $[10,12,13,14,16,21,22,24$, 27]. Consider an unstable SDS described by

$$
d x(t)=f(x(t), r(t), t) d t+g(x(t), r(t), t) d B(t),
$$

where the state $x(t)$ takes values in $\mathbb{R}^{n}$ and the mode $r(t)$ is a Markov chain taking values in a finite space $\mathbb{S}=\{1,2, \cdots, N\}, B(t)$ is a Brownian motion. In order to stabilize this given system, it is traditional to design a feedback control term $u(x(t), r(t), t)$ so that the controlled SDS (CSDS)

$$
d x(t)=[f(x(t), r(t), t)+u(x(t), r(t), t)] d t+g(x(t), r(t), t) d B(t)
$$

becomes stable. Due to the requirement of the continuous-time observations for the state $x(t)$, it is difficult to implement such a regular control. In practice, very high frequent state observations are used instead of continuous-time observations and hence the control cost is expensive. For the sake of saving costs and easy operation Mao [12] designed the feedback control based on the discrete-time observations (not necessarily high frequency), and developed the corresponding theory $[4,5,6]$ of deterministic systems to stochastic versions. That is, $u(x(\nu(t)), r(t), t)$ was designed, where $\nu(t):=[t / \tau] \tau$ with $\tau>0$ being the duration between two consecutive observations, such that the controlled system

$$
d x(t)=[f(x(t), r(t), t)+u(x(\nu(t)), r(t), t)] d t+g(x(t), r(t), t) d B(t),
$$

becomes stable in mean square. In the latter works $[14,27]$ much better lower bound on $\tau$ was obtained while other types of asymptotic stability were studied. However, from practical point of view it is sometimes necessary to degin the feedback control based on not only $x(\nu(t))$ but also $r(\nu(t))$ (see, e.g., [7, 24] for details). Due to the continuity of $x(t)$ the deviation of $x(t)-x(\nu(t))$ may be small as long as $\tau$ is sufficiently small. But the jump processes $r(\nu(t))$ and $r(t)$ may take different values in $\mathbb{S}$ even if $\tau$ is extremely small. This problem was tackled by $[9,24]$. In particular, using different method from [9, 24], Shao [21] obtained the stability in mean square for the linear controlled SDS based on the discrete-time observations of both the system state $x(\cdot)$ and the Markov mode $r(\cdot)$. Shao and $\mathrm{Xi}[22]$ went a further step to analyze the almost sure stability of the linear controlled SDS with the state-dependent regime switching.

Response lags are often required by most physical systems, and play a crucial role in the feedback loops [19]. Taking into account a time lag $\tau_{0}(>0)$ between the time when the observations for the state $(x(\nu(t)), r(\nu(t)))$ is made and the time when the feedback control reaches the system, it is more realistic to design the control dependent on the past discrete-time state pair $\left(x\left(\nu(t)-\tau_{0}\right), r\left(\nu(t)-\tau_{0}\right)\right)$. To our best knowledge, the existing papers in the literature on stabilisation problems by delay feedback control are based on the observations of only system state $x\left(t-\tau_{0}\right)$ or $x\left(\nu(t)-\tau_{0}\right)$, for examples, $[10,13,16,19]$. Our main aim in this paper is to design the feedback control $u\left(x\left(\nu(t)-\tau_{0}\right), r\left(\nu(t)-\tau_{0}\right), t\right)$ $\left(\tau_{0} \geq 0\right)$ so that the delay controlled SDS (DCSDS)

$$
d x(t)=\left[f(x(t), r(t), t)+u\left(x\left(\nu(t)-\tau_{0}\right), r\left(\nu(t)-\tau_{0}\right), t\right)\right] d t+g(x(t), r(t), t) d B(t)
$$


becomes stable in $p$ th moment, with probability one or in $H_{\infty}$.

Mathematically speaking, this paper uses the strong ergodicity theory of Markov chains and the asymptotic analysis techniques of stochastic functional differential equations (SFDEs), which are completely different from those used in the papers $[12,14,16$, $21,22,24,27]$ mentioned above. Various criteria on the uniform boundedness and different kinds of stability will be established for the DCSDS (1.3) when their coefficients are either quasi-linear or highly nonlinear. The main contributions of this paper are highlighted as follows.

- For the quasi-linear DCSDSs (1.3), we give sharp criteria on the uniform boundedness of the solution in infinite horizon as well as exponential stability in mean square. That is, by a feedback control satisfying a proposed condition, the solution will be uniformly bounded or exponentially stable in mean square, while it will be unbounded or unstable under a slightly weaker control. The explicit rates of the convergence and divergence are obtained.

- For the nonlinear DCSDSs (1.3), we give the criteria on the feedback control for the solution to be exponentially stable in $p$ th moment and probability one as well as in $H_{\infty}$. The sample and moment Lyapunov exponents are estimated, which describe the convergence speed that $x(t)$ tends to 0 in $p$ th moment and in sample path.

- The lower bound on $\tau^{*}$ is obtained explicitly so that the feedback control will stablise the given system as long as $\tau+\tau_{0}<\tau^{*}$. How the values of $\tau$ and $\tau_{0}$ affect the Lyapunov exponents is also investigated.

The rest of the paper is organised as follows. Section 2 begins with notations and preliminaries on the properties of the exact solutions. Section 3 focuses on the quasilinear DCSDS (1.3). The sharp criteria on boundedness (unboundedness) and stability (unstability) are established. The convergence and divergence rates are estimated. Section 4 pays attention to the stability analysis for the highly nonlinear DCSDSs (1.3). Under the conditions on the existence of the global regular solution of (1.1) and its boundedness in pth moment, it will be shown that the controlled system (1.3) preserves the boundedness. The lower bounds on both $\tau$ and $\tau_{0}$ are also given explicitly. The control principles for the controlled system (1.3) to be exponentially stable in $p$ th moment or in probability one or in $H_{\infty}$ are provided. Furthermore, the sample and moment Lyapunov exponents are estimated. in Section 5, an example with computer simulations is discussed to illustrate the theoretical results.

\section{Preliminary}

Throughout this paper, we use the following notations. If $A$ is a vector or matrix, its transpose is denoted by $A^{T}$ and its trace norm is denoted by $|A|=\sqrt{\operatorname{trace}\left(A^{T} A\right)}$. For vectors or matrixes $A$ and $B$ with compatible dimensions, $A B$ denotes the usual matrix multiplication. For any sequence $\left\{c_{i}\right\}_{1 \leq i \leq N}(N \in \mathbb{N})$, define $\hat{c}=\min _{1 \leq i \leq N} c_{i}$ and $\check{c}=$ $\max _{1 \leq i \leq N} c_{i}$. For any $a, b \in \mathbb{R}, a \vee b:=\max \{a, b\}$, and $a \wedge b:=\min \{a, b\}$.

Let $(\Omega, \mathcal{F}, \mathbb{P})$ be a complete probability space and $\mathbb{E}$ denote the expectation with respect to $\mathbb{P}$. Let $B(t)=\left(B_{1}(t), \cdots, B_{m}(t)\right)^{T}$ be an $m$-dimensional Brownian motion 
defined on the probability space. Let $r(t), t \geq 0$, be a right-continuous Markov chain on the probability space taking values in a finite state space $S=\{1,2, \cdots, N\}(N<\infty)$ with generator $\Gamma=\left(\gamma_{i j}\right)_{N \times N}$ given by

$$
\mathbb{P}\{r(t+\Delta)=j \mid r(t)=i\}= \begin{cases}\gamma_{i j} \Delta+o(\Delta) & \text { if } i \neq j \\ 1+\gamma_{i i} \Delta+o(\Delta) & \text { if } i=j\end{cases}
$$

where $\Delta \downarrow 0, o(\Delta)$ means $\lim _{\Delta \rightarrow 0} o(\Delta) / \Delta=0$. Here we assume $\Gamma$ is conservative (i.e. $-\gamma_{i i}=\sum_{j \neq i} \gamma_{i j}, \forall i \in \mathbb{S}$ ) and irreducible (i.e. the linear equations $\pi \Gamma=0$ and $\sum_{i=1}^{N} \pi_{i}=1$ has a unique solution $\pi=\left(\pi_{1}, \ldots, \pi_{N}\right) \in \mathbb{R}^{1 \times N}$ satisfying $\pi_{i}>0$ for each $\left.i \in \mathbb{S}\right)$. This solution is termed a stationary distribution. For a sequence $\left\{c_{i}\right\}_{1 \leq i \leq N}$, we will often write $c(i)=c_{i}$ and set $c=\left(c_{1}, \cdots, c_{N}\right)^{T}$, define $\pi c=\sum_{i=1}^{N} \pi_{i} c_{i}$. We assume that the Markov chain $r(\cdot)$ is independent of the Brownian motion $B(\cdot)$. Suppose $\left\{\mathcal{F}_{t}\right\}_{t \geq 0}$ is a filtration defined on this probability space satisfying the usual conditions (i.e., it is right continuous and $\mathcal{F}_{0}$ contains all $\mathbb{P}$-null sets) such that $B(t)$ and $r(t)$ are $\mathcal{F}_{t}$ adapted. Denote by $\mathcal{G}$ the $\sigma$-algebra generated by $\{r(t)\}_{0 \leq t<\infty}$. We also denote the conditional expectation $\mathbb{E}(\cdot \mid \mathcal{G})$ by $\mathbb{E}_{\mathcal{G}}(\cdot)$.

In the paper, we use the feedback control function with a simple form $u(x, i, t)=$ $-\alpha(i) x$ for $(x, i, t) \in \mathbb{R}^{n} \times \mathbb{S} \times \mathbb{R}_{+}$, where $\alpha(i)$ 's are all nonnegative constants. Suppose that the underlying system is described by the DCSDS (1.3) with the initial data

$$
x(t)=x_{0} \in \mathbb{R}^{n}, \quad r(t)=i_{0} \in \mathbb{S}, \quad-\tau_{0} \leq t \leq 0,
$$

while the coefficient functions $f: \mathbb{R}^{n} \times \mathbb{S} \times \mathbb{R}_{+} \rightarrow \mathbb{R}^{n}$ and $g: \mathbb{R}^{n} \times \mathbb{S} \times \mathbb{R}_{+} \rightarrow \mathbb{R}^{n \times m}$ satisfy the local Lipschitz condition, namely, for any real number $R>0$, there exists a positive constant $K_{R}$ such that

$$
|f(x, i, t)-f(\bar{x}, i, t)| \vee|g(x, i, t)-g(\bar{x}, i, t)| \leq K_{R}|x-\bar{x}|
$$

for all $x, \bar{x} \in \mathbb{R}^{n}$ with $|x| \vee|\bar{x}| \leq R$ and all $(i, t) \in \mathbb{S} \times \mathbb{R}_{+}$. It is well known that the local Lipschitz conditions of the coefficients only guarantee that the SDS (1.1) has a unique maximal local solution, which may explode to infinity at a finite time. To avoid such a possible explosion, we impose the following Khasminskii-type condition.

Assumption 1 Assume that there exist positive constants $A, C$, and $p \geq 2$ such that

$$
x^{T} f(x, i, t)+\frac{p-1}{2}|g(x, i, t)|^{2} \leq C+A|x|^{2}, \quad(x, i, t) \in \mathbb{R}^{n} \times \mathbb{S} \times \mathbb{R}_{+} .
$$

We prepare the regularity for the solutions of SDS (1.1) and DCSDS (1.3), respectively, as follows.

Lemma 2.1 [15, p. 93, Theorem 3.17] Under Assumption 1, the SDS (1.1) with the initial data $(x(0), r(0))=\left(x_{0}, i_{0}\right) \in \mathbb{R}^{n} \times \mathbb{S}$ has a unique global solution $x(t)$ on $[0, \infty)$.

In a similar way as $[15$, p. 89 , Theorem 3.13$]$ was proved, we can show:

Lemma 2.2 Under Assumption 1, the DCSDS (1.3) with the initial data (2.2) has a unique global solution $x(t)$ on $[0, \infty)$. 
In (1.3) the feedback control depends on the term $\alpha\left(r\left(\nu(t)-\tau_{0}\right)\right)$. To analyze the asymptotic property we need a number of new notations and recall some results from [3]. For any vector $\mu=\left(\mu_{1}, \ldots, \mu_{N}\right)^{T}$, any constant $l>0$, define

$$
\operatorname{diag}(\mu):=\operatorname{diag}\left(\mu_{1}, \ldots, \mu_{N}\right), \Gamma_{l, \mu}:=\Gamma-l \operatorname{diag}(\mu), \eta_{l, \mu}:=-\max _{\lambda \in \operatorname{spec}\left(\Gamma_{l, \mu}\right)} \operatorname{Re}(\lambda),
$$

where $\operatorname{spec}\left(\Gamma_{l, \mu}\right)$ and $\operatorname{Re}(\lambda)$ denote the spectrum of $\Gamma_{l, \mu}$ (i.e. the multiset of its eigenvalues) and the real part of $\lambda$, respectively.

Lemma 2.3 [3, Proposition 4.1, Proposition 4.2] For any $l>0$, there are two positive constants $K_{1}(l)$ and $K_{2}(l)$ such that for any $t>0$

$$
K_{1}(l) e^{-\eta_{l, \mu} t} \leq \mathbb{E}\left(e^{-l \int_{0}^{t} \mu(r(z)) d z}\right) \leq K_{2}(l) e^{-\eta_{l, \mu} t} .
$$

Moreover, if $\pi \mu>0$, there is a constant $\kappa_{\mu}>0$ such that $\eta_{l, \mu}>0$ for $l \in\left(0, \kappa_{\mu}\right)$ but $\eta_{l, \mu}<0$ for $l>\kappa_{\mu}$. Furthermore, if $\check{\mu} \geq 0, \kappa_{\mu}=\infty$; if $\check{\mu}<0, \kappa_{\mu} \in\left(0, \min _{i \in \mathbb{S}, \mu_{i}<0}\left\{\gamma_{i i} / \mu_{i}\right\}\right)$.

In order to obtain the dynamical behaviors of the solutions of DCSDS (1.3) we need to investigate the asymptotic properties of $\alpha\left(r\left(\nu(t)-\tau_{0}\right)\right)$. Firstly we redefine two Markov chains. Let $n_{0}=\left[\tau_{0} / \tau\right], \delta=\left(n_{0}+1\right) \tau-\tau_{0}, \tilde{r}(t):=r\left(t+\left(n_{0}+1\right) \tau\right)$ for $t \geq 0$, and $\tilde{r}_{n}:=\tilde{r}\left(n \tau-\tau_{0}\right)=r(n \tau+\delta)$ for any integer $n \geq 0$. Then $\left\{\tilde{r}_{n}\right\}_{n \geq 0}$ is a skeleton process of Markov chain $\{r(t)\}_{t \geq 0}$, which is a discrete-time homogeneous Markov chain on $\mathbb{S}$. Its transition probability matrix is $\left(P_{i j}\right)_{N \times N}$ with $P_{i j}=\mathbb{P}(r(\tau+\delta)=j \mid r(\delta)=i)$. By virtue of Lemma 2.3 we can obtain the following results.

Lemma 2.4 Let $h=\left(h_{1}, \cdots, h_{N}\right)^{T}$ such that $\pi \alpha>\pi h$. Then, for any constant $0<l<$ $\kappa_{\alpha-h}$, if $\tau<\bar{\tau}(l, \alpha-h)$, there are positive constants $K_{3}(l, \alpha-u)$ and $\zeta_{l, \alpha-h}^{\tau}$ defined by (2.13) such that for any $t \geq 0$

$$
\mathbb{E}\left(e^{l \int_{0}^{t}\left(h(\tilde{r}(z))-\alpha\left(\tilde{r}\left(\nu(z)-\tau_{0}\right)\right)\right) d z}\right) \leq K_{3}(l, \alpha-u) e^{-\zeta_{l, \alpha-h}^{\tau} t},
$$

where $\bar{\tau}=\bar{\tau}(l, \alpha-h)$ is the solution of the equation (in $\tau$ )

$$
\epsilon \max _{j \in \mathbb{S}}\left\{-\gamma_{j j}\right\}\left(e^{\frac{\tau l \check{\alpha}(1+\epsilon)}{\epsilon}}-1\right)=\eta_{l(1+\epsilon), \alpha-h}, \quad \epsilon:=\left[\left(\kappa_{\alpha-h}-l\right) / 2 l\right] \wedge 1 .
$$

Proof. By Hölder's inequality, we obtain that for any $0<l<\kappa_{\alpha-h}$ and the given $\epsilon>0$,

$$
\begin{aligned}
& \mathbb{E}\left(e^{l \int_{0}^{t}\left(h(\tilde{r}(z))-\alpha\left(\tilde{r}\left(\nu(z)-\tau_{0}\right)\right)\right) d z}\right) \\
& \leq\left(\mathbb{E} e^{(1+\epsilon) l \int_{0}^{t}(h(\tilde{r}(z))-\alpha(\tilde{r}(z))) d z}\right)^{\frac{1}{1+\epsilon}}\left(\mathbb{E} e^{\frac{l(1+\epsilon)}{\epsilon} \int_{0}^{t}\left(\alpha(\tilde{r}(z))-\alpha\left(\tilde{r}\left(\nu(z)-\tau_{0}\right)\right)\right) d z}\right)^{\frac{\epsilon}{1+\epsilon}} .
\end{aligned}
$$

By virtue of Lemma 2.3 we know that

$$
\left(\mathbb{E} e^{(1+\epsilon) l \int_{0}^{t}(h(\tilde{r}(z))-\alpha(\tilde{r}(z))) d z}\right)^{\frac{1}{1+\epsilon}} \leq\left[K_{2}((1+\epsilon) l)\right]^{\frac{1}{1+\epsilon}} e^{-\frac{\eta_{l(1+\epsilon), \alpha-h}}{1+\epsilon} t} .
$$


Recalling the fact that $\alpha$ is a nonnegative vector, we drive

$$
\begin{aligned}
-\int_{0}^{t} \alpha\left(\tilde{r}\left(\nu(z)-\tau_{0}\right)\right) d z & =-\int_{-\left(n_{0}+1\right) \tau}^{t-\left(n_{0}+1\right) \tau} \alpha\left(\tilde{r}\left(\nu\left(z+\left(n_{0}+1\right) \tau\right)-\tau_{0}\right)\right) d z \\
& \leq-\int_{0}^{t} \alpha(\tilde{r}(\nu(z)+\delta)) d z+\int_{t-\left(n_{0}+1\right) \tau}^{t} \alpha(\tilde{r}(\nu(z)+\delta)) d z \\
& \leq-\int_{0}^{t} \alpha(\tilde{r}(\nu(z)+\delta)) d z+\left(n_{0}+1\right) \tau \check{\alpha} .
\end{aligned}
$$

This implies that

$$
\begin{aligned}
& \mathbb{E} e^{\frac{l(1+\epsilon)}{\epsilon} \int_{0}^{t}\left(\alpha(\tilde{r}(z))-\alpha\left(\tilde{r}\left(\nu(z)-\tau_{0}\right)\right)\right) d z} \\
& \leq e^{\frac{(1+\epsilon)}{\epsilon} l\left(n_{0}+1\right) \tau \check{\alpha}} \mathbb{E} e^{\frac{l(1+\epsilon)}{\epsilon} \int_{0}^{t}(\alpha(\tilde{r}(z))-\alpha(\tilde{r}(\nu(z)+\delta))) d z} \\
& \leq e^{\frac{(1+\epsilon)}{\epsilon} l\left(n_{0}+1\right) \tau \check{\alpha}} \mathbb{E} e^{\frac{l(1+\epsilon)}{\epsilon} \sum_{i=0}^{[t / \tau]} \int_{i \tau}^{(i+1) \tau}|\alpha(\tilde{r}(z))-\alpha(\tilde{r}(i \tau+\delta))| d z} \\
& =e^{\frac{(1+\epsilon)}{\epsilon} l\left(n_{0}+1\right) \tau \check{\alpha}} \mathbb{E}\left(\prod_{i=0}^{[t / \tau]} e^{\frac{l(1+\epsilon)}{\epsilon} \int_{i \tau}^{(i+1) \tau}|\alpha(\tilde{r}(z))-\alpha(\tilde{r}(i \tau+\delta))| d z}\right) .
\end{aligned}
$$

For any nonnegative integer $i$, the Jensen inequality shows

$$
\begin{aligned}
& \mathbb{E}\left(e^{\frac{l(1+\epsilon)}{\epsilon} \int_{i \tau}^{(i+1) \tau}|\alpha(\tilde{r}(z))-\alpha(\tilde{r}(\nu(z)+\delta))| d z} \mid \tilde{r}(i \tau)\right) \\
& \leq \mathbb{E}\left(\frac{1}{\tau} \int_{i \tau}^{(i+1) \tau} e^{\frac{\tau l(1+\epsilon)}{\epsilon}|\alpha(\tilde{r}(z))-\alpha(\tilde{r}(\nu(z)+\delta))|} d z \mid \tilde{r}(i \tau)\right) \\
& =\frac{1}{\tau} \sum_{j=1}^{N} I_{\{\tilde{r}(i \tau)=j\}} \int_{i \tau}^{(i+1) \tau} \mathbb{E}\left(e^{\frac{\tau l(1+\epsilon)}{\epsilon}|\alpha(\tilde{r}(z))-\alpha(\tilde{r}(i \tau+\delta))|} \mid \tilde{r}(i \tau)=j\right) d z .
\end{aligned}
$$

It is known that the waiting time for the next jump of the Markov chain $\tilde{r}(\cdot)$ from current state $j$ obeys the exponential distribution with parameter $-\gamma_{j j}$ (see, e.g., [1, p. 16, Proposition 2.8]). Thus, noticing that $1-e^{-x} \leq x$ for $x \geq 0$, we have

$$
\begin{aligned}
\mathbb{E}( & \left.e^{\frac{l(1+\epsilon)}{\epsilon} \int_{i \tau}^{(i+1) \tau}|\alpha(\tilde{r}(z))-\alpha(\tilde{r}(i \tau+\delta))| d z} \mid \tilde{r}(i \tau)\right) \\
= & \frac{1}{\tau} \sum_{j=1}^{N} I_{\{\tilde{r}(i \tau)=j\}} \int_{i \tau}^{(i+1) \tau} \mathbb{E}\left(I_{\{\tilde{r}(v)=j, \forall v \in[i \tau,(i+1) \tau]\}}\right. \\
& \left.\quad+I_{\{\exists v \in[i \tau,(i+1) \tau], \tilde{r}(v) \neq j\}} e^{\frac{\tau l(1+\epsilon)}{\epsilon}|\alpha(\tilde{r}(z))-\alpha(\tilde{r}(i \tau+\delta))|} \mid \tilde{r}(i \tau)=j\right) d z \\
\leq & \frac{1}{\tau} \sum_{j=1}^{N} I_{\{\tilde{r}(i \tau)=j\}} \int_{i \tau}^{(i+1) \tau}(\mathbb{P}(\tilde{r}(v)=j, \forall v \in[i \tau,(i+1) \tau] \mid \tilde{r}(i \tau)=j) \\
& \left.+e^{\frac{\tau l \tilde{\alpha}(1+\epsilon)}{\epsilon}} \mathbb{P}(\exists v \in[i \tau,(i+1) \tau], \tilde{r}(v) \neq j \mid \tilde{r}(i \tau)=j)\right) d z \\
= & \frac{1}{\tau} \sum_{j=1}^{N} I_{\{\tilde{r}(i \tau)=j\}} \int_{i \tau}^{(i+1) \tau}\left(e^{\gamma_{j j} \tau}+e^{\frac{\tau l \tilde{\alpha}(1+\epsilon)}{\epsilon}}\left(1-e^{\gamma_{j j} \tau}\right)\right) d z \\
= & \sum_{j=1}^{N} I_{\{\tilde{r}(i \tau)=j\}}\left(1+\left(e^{\frac{\tau l \tilde{\alpha}(1+\epsilon)}{\epsilon}}-1\right)\left(1-e^{\gamma_{j j} \tau}\right)\right) \leq 1+\tau \Lambda_{\tau}(l),
\end{aligned}
$$


where

$$
\Lambda_{\tau}(l):=\max _{j \in \mathbb{S}}\left\{-\gamma_{j j}\right\}\left(e^{\frac{\tau l \check{\alpha}(1+\epsilon)}{\epsilon}}-1\right) .
$$

Inserting this inequality into (2.9) yields that

$$
\begin{aligned}
& \mathbb{E} e^{\frac{l(1+\epsilon)}{\epsilon} \int_{0}^{t} \alpha(\tilde{r}(z))-\alpha\left(\tilde{r}\left(\nu(z)-\tau_{0}\right)\right) d z} \\
& \leq e^{\frac{(1+\epsilon)}{\epsilon} l\left(n_{0}+1\right) \tau \check{\alpha}} \mathbb{E}\left[\mathbb{E}\left(\prod_{i=0}^{[t / \tau]} e^{\frac{l(1+\epsilon)}{\epsilon} \int_{i \tau}^{(i+1) \tau}|\alpha(\tilde{r}(z))-\alpha(\tilde{r}(i \tau+\delta))| d z} \mid \tilde{r}([t / \tau] \tau)\right)\right] \\
& \leq e^{\frac{(1+\epsilon)}{\epsilon} l\left(n_{0}+1\right) \tau \check{\alpha}} \mathbb{E}\left[\prod_{i=0}^{[t / \tau]-1} e^{\frac{l(1+\epsilon)}{\epsilon} \int_{i \tau}^{(i+1) \tau}|\alpha(\tilde{r}(z))-\alpha(\tilde{r}(i \tau+\delta))| d z}\right. \\
& \left.\quad \times \mathbb{E}\left(e^{\frac{l(1+\epsilon)}{\epsilon} \int_{[t / \tau] \tau}^{([t / \tau]+1) \tau}|\alpha(\tilde{r}(z))-\alpha(\tilde{r}([t / \tau] \tau+\delta))| d z} \mid \tilde{r}([t / \tau] \tau)\right)\right] \\
& \leq e^{\frac{(1+\epsilon)}{\epsilon} l\left(n_{0}+1\right) \tau \check{\alpha}}\left(1+\tau \Lambda_{\tau}(l)\right)^{[t / \tau]+1} \\
& \leq e^{\frac{(1+\epsilon)}{\epsilon} l\left(n_{0}+1\right) \tau \check{\alpha}} e^{([t / \tau]+1) \tau \Lambda_{\tau}(l)} \\
& \leq e^{\frac{(1+\epsilon)}{\epsilon} l\left(n_{0}+1\right) \tau \check{\alpha}+\tau \Lambda_{\tau}(l)} e^{t \Lambda_{\tau}(l)} .
\end{aligned}
$$

Thus, inserting (2.7) and (2.11) into (2.6) we obtain

$$
\mathbb{E}\left(e^{l \int_{0}^{t}\left(h(\tilde{r}(z))-\alpha\left(\tilde{r}\left(\nu(z)-\tau_{0}\right)\right)\right) d z}\right) \leq K_{3}(l, \alpha-h) e^{-\zeta_{l, \alpha-h}^{\tau} t},
$$

where

$$
\begin{aligned}
K_{3}(l, \alpha-h) & :=\left[K_{2}((1+\epsilon) l)\right]^{\frac{1}{1+\epsilon}} e^{\frac{\epsilon \tau \Lambda_{\tau}(l)}{1+\epsilon}+l\left(n_{0}+1\right) \tau \check{\alpha}}, \\
\zeta_{l, \alpha-h}^{\tau} & :=\frac{\eta_{l(1+\epsilon), \alpha-h}-\epsilon \Lambda_{\tau}(l)}{1+\epsilon} .
\end{aligned}
$$

Using $\tau<\bar{\tau}(l, \alpha-h)$ and the definition of $\Lambda_{\tau}(l)$, we know that $\zeta_{l, \alpha-h}^{\tau}>0$. Therefore, the required assertion follows.

Lemma 2.5 Let $h=\left(h_{1}, \cdots, h_{N}\right)^{T}$ such that $\pi \alpha<\pi h$. Then, for any constants $l>0$ and $0<\epsilon<\pi h-\pi \alpha$, there is a constant $T>0$ such that for any $s \in[0, \infty)$

$$
\mathbb{E}\left(e^{l \int_{s}^{s+t}\left(h(r(z))-\alpha\left(r\left(\nu(z)-\tau_{0}\right)\right)\right) d z}\right) \geq K_{1}(l, \alpha-h) e^{l(\pi h-\pi \alpha-\epsilon) t}, \quad t \geq T .
$$

where $K_{1}(l, \alpha-h):=e^{-l\left(2 \tau+\tau_{0}\right)\left(\check{\alpha}+2 \max _{i \in \mathbb{S}}|h(i)|\right)}$.

Proof. Since Markov chains $\{\tilde{r}(t)\}_{t \geq 0}$ and $\left\{\tilde{r}_{n}\right\}_{n \geq 0}$ are ergodic and has the same stationary distribution $\left(\pi_{1}, \ldots, \pi_{N}\right)$, by the strong ergodic theorem and the boundedness of $h(\cdot)$ and $\alpha(\cdot)$, we have

$$
\begin{aligned}
& \lim _{t \rightarrow \infty} \frac{1}{t} \int_{0}^{t}\left(h(\tilde{r}(z))-\alpha\left(\tilde{r}\left(\nu(z)-\tau_{0}\right)\right)\right) d z \\
& =\pi h-\lim _{t \rightarrow \infty} \frac{1}{t}\left[\tau \sum_{i=0}^{[t / \tau]-1} \alpha\left(\tilde{r}_{i}\right)+\alpha\left(\tilde{r}_{[t / \tau]}\right)(t-\nu(t))\right] \\
& =\pi h-\lim _{t \rightarrow \infty}\left(\frac{\nu(t)}{t} \cdot \frac{\sum_{i=0}^{[t / \tau]-1} \alpha\left(\tilde{r}_{i}\right)}{[t / \tau]}+\frac{\alpha\left(\tilde{r}_{[t / \tau]}\right)(t-\nu(t))}{t}\right) \\
& =\pi h-\pi \alpha, \quad \mathbb{P}_{2}-\text { a.s. }
\end{aligned}
$$


If $\pi \alpha<\pi h$, for any given constants $l>0$ and $0<\epsilon<\pi h-\pi \alpha$, one observes from (2.15) that

$$
\lim _{t \rightarrow \infty} \frac{l}{t} \int_{0}^{t}\left(h(\tilde{r}(z))-\alpha\left(\tilde{r}\left(\nu(z)-\tau_{0}\right)\right)-\pi h+\pi \alpha+\epsilon\right) d z=l \epsilon>0, \quad \mathbb{P}_{2}-\text { a.s. }
$$

which implies that

$$
\lim _{t \rightarrow \infty} e^{l \int_{0}^{t}\left(h(\tilde{r}(z))-\alpha\left(\tilde{r}\left(\nu(z)-\tau_{0}\right)\right)-\pi h+\pi \alpha+\epsilon\right) d z}=\infty \mathbb{P}_{2}-\text { a.S. }
$$

By virtue of the Fatou lemma (see, e.g. [23, p.187, Theorem 2]) we have

$$
\liminf _{t \rightarrow \infty} \mathbb{E}\left(e^{l \int_{0}^{t}\left(h(\tilde{r}(z))-\alpha\left(\tilde{r}\left(\nu(z)-\tau_{0}\right)\right)-\pi h+\pi \alpha+\epsilon\right) d z}\right)=\infty .
$$

Hence, there is a constant $T>0$ such that

$$
\mathbb{E}\left(e^{l \int_{0}^{t}\left(h(\tilde{r}(z))-\alpha\left(\tilde{r}\left(\nu(z)-\tau_{0}\right)\right)\right) d z}\right) \geq e^{l(\pi h-\pi \alpha-\epsilon) t}, \quad t \geq T
$$

holds. Due to the homogeneousness of Markov chains $\{\tilde{r}(t)\}_{t \geq 0}$ and $\left\{\tilde{r}_{n}\right\}_{n \in \mathbb{N}}$, we know that for any $s>0$

$$
\begin{aligned}
& \mathbb{E}\left(e^{l \int_{0}^{t}\left(h(\tilde{r}(z+s))-\alpha\left(\tilde{r}\left(\nu(z+s)-\tau_{0}\right)\right)\right) d z}\right) \\
& =\mathbb{E}\left(\mathbb{E}\left(e^{l \int_{0}^{t}\left(h(\tilde{r}(z+s))-\alpha\left(\tilde{r}\left(\nu(z+s)-\tau_{0}\right)\right)\right) d z} \mid \tilde{r}(\nu(s))\right)\right) \\
& =\mathbb{E}\left(\mathbb{E}\left(e^{l \int_{0}^{t}\left(h(\tilde{r}(z+s-\nu(s)))-\alpha\left(\tilde{r}\left(\nu(z+s)-\nu(s)-\tau_{0}\right)\right)\right) d z} \mid \tilde{r}(0)\right)\right) \\
& =\mathbb{E}\left(e^{l \int_{0}^{t}\left(h\left(\tilde{r}\left(z+\delta_{s}\right)-\alpha\left(\tilde{r}\left(\nu\left(z+\delta_{s}\right)-\tau_{0}\right)\right)\right) d z\right.}\right)
\end{aligned}
$$

where $\delta_{s}:=s-\nu(s)$ for any $s \geq 0$. From $0 \leq \delta_{s}<\tau$, one observes that

$$
\begin{aligned}
& \int_{0}^{t}\left(h\left(\tilde{r}\left(z+\delta_{s}\right)-\alpha\left(\tilde{r}\left(\nu\left(z+\delta_{s}\right)-\tau_{0}\right)\right)\right) d z\right. \\
& =\int_{\delta_{s}}^{t+\delta_{s}}\left(h(\tilde{r}(z))-\alpha\left(\tilde{r}\left(\nu(z)-\tau_{0}\right)\right)\right) d z \\
& =\int_{0}^{t}\left(h(\tilde{r}(z))-\alpha\left(\tilde{r}\left(\nu(z)-\tau_{0}\right)\right)\right) d z+\int_{t}^{t+\delta_{s}}\left(h(\tilde{r}(z))-\alpha\left(\tilde{r}\left(\nu(z)-\tau_{0}\right)\right)\right) d z \\
& \quad-\int_{0}^{\delta_{s}}\left(h(\tilde{r}(z))-\alpha\left(\tilde{r}\left(\nu(z)-\tau_{0}\right)\right)\right) d z \\
& \geq \int_{0}^{t}\left(h(\tilde{r}(z))-\alpha\left(\tilde{r}\left(\nu(z)-\tau_{0}\right)\right)\right) d z-2 \tau \max _{i \in \mathbb{S}}|h(i)|-\tau \check{\alpha} .
\end{aligned}
$$

Inserting (2.18) into (2.17), then using (2.16), we obtain that for any $s \geq 0$

$$
\begin{aligned}
& \mathbb{E}\left(e^{l \int_{0}^{t}\left(h(\tilde{r}(z+s))-\alpha\left(\tilde{r}\left(\nu(z+s)-\tau_{0}\right)\right)\right) d z}\right) \\
& \geq e^{-\tau l\left(\check{\alpha}+2 \max _{i \in \mathbb{S}}|h(i)|\right)} \mathbb{E}\left(e^{l \int_{0}^{t}\left(h(\tilde{r}(z))-\alpha\left(\tilde{r}\left(\nu(z)-\tau_{0}\right)\right)\right) d z}\right) \\
& \geq e^{-\tau l\left(\check{\alpha}+2 \max _{i \in \mathbb{S}}|h(i)|\right)} e^{l(\pi h-\pi \alpha-\epsilon) t}, \quad t \geq T .
\end{aligned}
$$


This, together with the definition of $\tilde{r}(t)$, implies that for any $s \in\left[\left(n_{0}+1\right) \tau, \infty\right)$

$$
\mathbb{E}\left(e^{l \int_{s}^{s+t}\left(h(r(z))-\alpha\left(r\left(\nu(z)-\tau_{0}\right)\right)\right) d z}\right) \geq e^{-l \tau\left(\check{\alpha}+2 \max _{i \in \mathbb{S}}|h(i)|\right)} e^{l(\pi h-\pi \alpha-\epsilon) t}, \quad t \geq T .
$$

By the similar way as (2.18) we know that for any $s \in\left[0,\left(n_{0}+1\right) \tau\right)$,

$$
\begin{aligned}
& \left.\int_{s}^{s+t}\left(h(r(z))-\alpha\left(r(\nu(z))-\tau_{0}\right)\right)\right) d z \\
& \geq \int_{\left(n_{0}+1\right) \tau}^{t+\left(n_{0}+1\right) \tau}\left(h(r(z))-\alpha\left(r\left(\nu(z)-\tau_{0}\right)\right)\right) d z-\left(n_{0}+1\right) \tau\left(\check{\alpha}+2 \max _{i \in \mathbb{S}}|h(i)|\right) .
\end{aligned}
$$

This together with (2.20) implies that for any $s \in[0, \infty)$

$$
\mathbb{E}\left(e^{l \int_{s}^{s+t}\left(h(r(z))-\alpha\left(r\left(\nu(z)-\tau_{0}\right)\right)\right) d z}\right) \geq e^{-l \tau\left(n_{0}+2\right)\left(\check{\alpha}+2 \max _{i \in \mathbb{S}}|h(i)|\right)} e^{l(\pi h-\pi \alpha-\epsilon) t}, \quad t \geq T .
$$

The required assertion (2.14) follows.

In a similar way as Lemma 2.5 was proved, we can use the homogeneousness of $\tilde{r}(\cdot)$ and Lemma 2.4 to show the following result.

Lemma 2.6 Let $h=\left(h_{1}, \cdots, h_{N}\right)^{T}$ such that $\pi \alpha>\pi h$. For any constant $0<l<\kappa_{\alpha-h}$, if $\tau<\bar{\tau}(l, \alpha-h)$, there is a positive constant $K_{2}(l, \alpha-u)$ such that for any $s \geq 0$

$$
\mathbb{E}\left(e^{l \int_{s}^{s+t}\left(h(r(z))-\alpha\left(r\left(\nu(z)-\tau_{0}\right)\right)\right) d z}\right) \leq K_{2}(l, \alpha-u) e^{-\zeta_{l, \alpha-h}^{\tau} t}, \quad t \geq 0,
$$

where $K_{2}(l, \alpha-u):=K_{3}(l, \alpha-u) e^{l\left(2 \tau+\tau_{0}\right)\left(\check{\alpha}+2 \max _{i \in \mathbb{S}}|h(i)|\right)}, \bar{\tau}(l, \alpha-h)$ and $\zeta_{l, \alpha-h}^{\tau}$ are given in Lemma 2.4.

In order to deal with the asymptotic properties of the SFDE (1.3), we begin with defining two segments $\bar{x}_{t}(s):=\left\{x(t+s):-2\left(\tau+\tau_{0}\right) \leq s \leq 0\right\}$ and $\bar{r}_{t}(s):=\{r(t+s)$ : $\left.-2\left(\tau+\tau_{0}\right) \leq s \leq 0\right\}$ for $t \geq 0$. In order for $\bar{x}_{t}$ and $\bar{r}_{t}$ to be well defined on $0 \leq t<2\left(\tau+\tau_{0}\right)$, we let $x(s)=x_{0}$ and $r(s)=i_{0}$ for $s \in\left[-2\left(\tau+\tau_{0}\right),-\tau_{0}\right)$. Moreover, we enlarge the definition domains of $f, g$ and $u$. For any $(x, i, t) \in \mathbb{R}^{n} \times \mathbb{S} \times\left[-2\left(\tau+\tau_{0}\right), 0\right)$, let $f(x, i, t)=f(x, i, 0)$, $g(x, i, t)=g(x, i, 0), u(x, i, t)=u(x, i, 0)$. In order to control the derivation from time delay in mean square, i.e. the value of $\mathbb{E}_{\mathcal{G}}\left|x(t)-x\left(\nu(t)-\tau_{0}\right)\right|^{2}$, we define an auxiliary functional

$$
\begin{gathered}
I\left(\bar{x}_{t}, \bar{r}_{t}, t\right):=\int_{-\left(\tau+\tau_{0}\right)}^{0} \int_{t+s}^{t}\left[\left(\tau+\tau_{0}\right)\left|f(x(z), r(z), z)-\alpha\left(r\left(\nu(z)-\tau_{0}\right)\right) x\left(\nu(z)-\tau_{0}\right)\right|^{2}\right. \\
\left.+|g(x(z), r(z), z)|^{2}\right] d z d s .
\end{gathered}
$$

For simplicity we let $I(t)=I\left(\bar{x}_{t}, \bar{r}_{t}, t\right)$. A direct calculation arrives at

$$
d I(t)=J_{1}(t) d t-J_{2}(t) d t,
$$

where

$$
\begin{aligned}
& J_{1}(t)=\left(\tau+\tau_{0}\right)\left[\left(\tau+\tau_{0}\right)\left|f(x(t), r(t), t)-\alpha\left(r\left(\nu(t)-\tau_{0}\right)\right) x\left(\nu(t)-\tau_{0}\right)\right|^{2}\right. \\
& \left.+|g(x(t), r(t), t)|^{2}\right] \\
& J_{2}(t)=\int_{-\left(\tau+\tau_{0}\right)}^{0}\left[\left(\tau+\tau_{0}\right) \mid f(x(t+s), r(t+s), t+s)\right. \\
& \left.-\left.\alpha\left(r\left(\nu(t+s)-\tau_{0}\right)\right) x\left(\nu(t+s)-\tau_{0}\right)\right|^{2}+|g(x(t+s), r(t+s), t+s)|^{2}\right] d s .
\end{aligned}
$$


By changing the integration order, we get

$$
I(t) \leq\left(\tau+\tau_{0}\right) J_{2}(t) .
$$

Using the Hölder inequality and the Itô isometry formula we go a further step to obtain

$$
\begin{aligned}
& \mathbb{E}_{\mathcal{G}}\left|x(t)-x\left(\nu(t)-\tau_{0}\right)\right|^{2} \\
& =\mathbb{E}_{\mathcal{G}} \mid \int_{\nu(t)-\tau_{0}}^{t}\left[f(x(s), r(s), s)-\alpha\left(r\left(\nu(s)-\tau_{0}\right)\right) x\left(\nu(s)-\tau_{0}\right)\right] d s \\
& \quad+\left.g(x(s), r(s), s) d B(s)\right|^{2} \\
& \quad \leq 2 \mathbb{E}_{\mathcal{G}} \int_{t-\left(\tau+\tau_{0}\right)}^{t}\left(\tau+\tau_{0}\right)\left|f(x(s), r(s), s)-\alpha\left(r\left(\nu(s)-\tau_{0}\right)\right) x\left(\nu(s)-\tau_{0}\right)\right|^{2} d s \\
& \quad+2 \mathbb{E}_{\mathcal{G}} \int_{t-\left(\tau+\tau_{0}\right)}^{t}|g(x(s), r(s), s)|^{2} d s=2 \mathbb{E}_{\mathcal{G}}\left[J_{2}(t)\right] .
\end{aligned}
$$

\section{Control of Quasi-linear Systems}

This section pays attention to design the control functions for the solutions of quasi-linear SDSs to be bounded in the infinite time horizon, and exponentially stable in $p$ th moment and in $\mathbb{P}$-a.s.

\subsection{Boundedness Control}

As is well-known that the unique solution of a linear SDS exists globally on $[0, \infty)$, and its $p$ th moment is finite on any finite time interval $[0, T]$. However its $p$ th moment may be unbounded in infinite horizon $[0, \infty)$. So it is necessary to design the control function $u\left(x\left(\nu(t)-\tau_{0}\right), r\left(\nu(t)-\tau_{0}\right), t\right)$ such that the solution of the controlled system (1.3) is bounded in mean square in $[0, \infty)$. To be precise we state the hypothesis of the linear growth condition on the coefficients.

Assumption 2 There exist positive constants $\bar{K}, D_{i}$ and $E_{i}$ such that

$$
|f(x, i, t)| \vee|g(x, i, t)| \leq \bar{K}(1+|x|)
$$

and

$$
x^{T} f(x, i, t)+\frac{1}{2}|g(x, i, t)|^{2} \leq E_{i}+D_{i}|x|^{2}
$$

hold for all $(x, i, t) \in \mathbb{R}^{n} \times \mathbb{S} \times \mathbb{R}_{+}$.

Theorem 3.1 Let Assumption 2 hold and set $D=\left(D_{1}, \cdots, D_{N}\right)^{T}$. Assume that $\pi \alpha>$ $\pi D$ and $\kappa_{\alpha-D}>2$. If $\tau \leq \tilde{\tau}:=\bar{\tau}(2, \alpha-D) / 2, \tau+\tau_{0} \leq \tau_{1}^{*}:=y_{1} \wedge y_{2}$, then the solution of DCSDS (1.3) with the initial condition (2.2) has the property that

$$
\sup _{0 \leq t<\infty} \mathbb{E}|x(t)|^{2}<\infty
$$

where $\bar{\tau}(\cdot, \cdot)$ is given as the solution of equation $(2.5), y_{1}$ and $y_{2}$ are the positive solutions of equations

$$
\bar{\beta}_{1}(y)=\frac{\zeta^{2}}{2\left(8 \check{\alpha}^{2}+\zeta^{2}\right)}, \quad \bar{\beta}_{2}(y)=\frac{\zeta^{2}}{8 \check{\alpha}^{2}+\zeta^{2}},
$$


respectively. Here we write $\zeta=\zeta_{2, \alpha-h}^{\tilde{\tau}}$ for short, $\bar{\beta}_{1}(\cdot), \bar{\beta}_{2}(\cdot)$ are defined by (3.6) and (3.12) below, respectively.

Proof. Fix $0<\tau \leq \tilde{\tau}$ and $0<\tau+\tau_{0} \leq \tau_{1}^{*}$. Using (3.1) we compute $I(t)$ and $J_{1}(t)$ defined by $(2.24)$ and $(2.26)$

$$
\begin{aligned}
J_{1}(t) \leq 2(\tau & \left.+\tau_{0}\right)\left[\left(2\left(\tau+\tau_{0}\right)\left(\bar{K}^{2}+\check{\alpha}^{2}\right)+\bar{K}^{2}\right)|x(t)|^{2}\right. \\
& \left.+2 \check{\alpha}^{2}\left(\tau+\tau_{0}\right)\left|x(t)-x\left(\nu(t)-\tau_{0}\right)\right|^{2}+2 \bar{K}^{2}\left(\tau+\tau_{0}\right)+\bar{K}^{2}\right] .
\end{aligned}
$$

Inserting (3.4) into (2.25) yields

$$
d I(t) \leq\left(\bar{\beta}_{1}\left(\tau+\tau_{0}\right)|x(t)|^{2}+\iota\left(\tau+\tau_{0}\right)\left|x(t)-x\left(\nu(t)-\tau_{0}\right)\right|^{2}+\bar{\beta}_{1}\left(\tau+\tau_{0}\right)-J_{2}(t)\right) d t
$$

where for any $y \geq 0$,

$$
\bar{\beta}_{1}(y):=2 y\left[2 y\left(\bar{K}^{2}+\check{\alpha}^{2}\right)+\bar{K}^{2}\right], \quad \iota(y):=4 \check{\alpha}^{2} y^{2} .
$$

Using the Itô formula and the elementary inequality, by (3.2), for $\zeta>0$, we derive

$$
\begin{aligned}
d|x(t)|^{2} \leq[2 \check{E}+ & \left(2 D(r(t))-2 \alpha\left(r\left(\nu(t)-\tau_{0}\right)\right)+\frac{\zeta}{2}\right)|x(t)|^{2} \\
& \left.+\frac{2 \check{\alpha}^{2}}{\zeta}\left|x(t)-x\left(\nu(t)-\tau_{0}\right)\right|^{2}\right] d t+2 x^{T}(t) g(x(t), r(t), t) d B(t) .
\end{aligned}
$$

Define $V\left(\bar{x}_{t}, \bar{r}_{t}, t\right)=\left(|x(t)|^{2}+\eta I(t)\right) e^{-2 \int_{0}^{t} \phi(s) d s}$, where $\phi(s):=D(r(s))-\alpha\left(r\left(\nu(s)-\tau_{0}\right)\right)+$ $3 \zeta / 8, \eta:=\zeta / 2+4 \check{\alpha}^{2} / \zeta$. Since $\phi(s)$ has only a finite number of jumps in any finite interval $[0, t], \int_{0}^{t} \phi(s) d s$ is differentiable. It follows from (3.5) and (3.7) that for any $t \geq 0$,

$$
\begin{aligned}
d V\left(\bar{x}_{t}, \bar{r}_{t}, t\right) \leq & e^{-2 \int_{0}^{t} \phi(s) d s}\left[-2 \eta \phi(t) I(t)-\left(\frac{\zeta}{4}-\eta \bar{\beta}_{1}\left(\tau+\tau_{0}\right)\right)|x(s)|^{2}\right. \\
+ & \left(\frac{2 \check{\alpha}^{2}}{\zeta}+\eta \iota\left(\tau+\tau_{0}\right)\right)\left|x(s)-x\left(\nu(s)-\tau_{0}\right)\right|^{2}+\left(2 \check{E}+\eta \bar{\beta}_{1}\left(\tau+\tau_{0}\right)\right) \\
& \left.-\eta J_{2}(t)\right] d t+2 e^{-2 \int_{0}^{t} \phi(s) d s} x^{T}(t) g(x(t), r(t), t) d B(t) .
\end{aligned}
$$

Due to the increasing property of $\bar{\beta}_{1}(y)$ in $y>0$, we see $\eta \bar{\beta}_{1}\left(\tau+\tau_{0}\right) \leq \zeta / 4$. One observes from (2.28) that

$$
-2 \eta \phi(t) I(t) \leq 2 \eta \check{\alpha} I(t) \leq 2 \eta \check{\alpha}\left(\tau+\tau_{0}\right) J_{2}(t) .
$$

These, together with (3.8), imply

$$
\begin{aligned}
d V\left(\bar{x}_{t}, \bar{r}_{t}, t\right) \leq & e^{-2 \int_{0}^{t} \phi(s) d s}\left[\left(\frac{2 \check{\alpha}^{2}}{\zeta}+\eta \iota\left(\tau+\tau_{0}\right)\right)\left|x(s)-x\left(\nu(s)-\tau_{0}\right)\right|^{2}\right. \\
& \left.+\left(2 \check{E}+\frac{\zeta}{4}\right)-\left(\eta-2 \eta \check{\alpha}\left(\tau+\tau_{0}\right)\right) J_{2}(t)\right] d t \\
& +2 e^{-2 \int_{0}^{t} \phi(s) d s} x^{T}(t) g(x(t), r(t), t) d B(t) .
\end{aligned}
$$


Integrating (3.10) on both sides and then taking the conditional expectation with respect to $\mathcal{G}$ and using $(2.29)$, we arrive at

$$
\begin{aligned}
\mathbb{E}_{\mathcal{G}}\left(e^{-2 \int_{0}^{t} \phi(s) d s}|x(t)|^{2}\right)+\eta \mathbb{E}_{\mathcal{G}}\left(e^{-2 \int_{0}^{t} \phi(s) d s} I(t)\right) \\
\leq\left|x_{0}\right|^{2}+\eta I(0)+\left(2 \check{E}+\frac{\zeta}{4}\right) \int_{0}^{t} e^{-2 \int_{0}^{s} \phi(z) d z} d s \\
-\left(\eta-\frac{4 \check{\alpha}^{2}}{\zeta}-\eta \bar{\beta}_{2}\left(\tau+\tau_{0}\right)\right) \int_{0}^{t} e^{-2 \int_{0}^{s} \phi(z) d z} \mathbb{E}_{\mathcal{G}}\left[J_{2}(s)\right] d s
\end{aligned}
$$

where for any constant $y \geq 0$,

$$
\bar{\beta}_{2}(y):=2 \iota(y)+2 y \check{\alpha}=4 \check{\alpha} y(\check{\alpha} y+1) .
$$

Due to the increasing property of $\bar{\beta}_{2}(y)$ in $y>0$ as well as by the definition of $\eta$, we see that $\eta \bar{\beta}_{2}\left(\tau+\tau_{0}\right) \leq \zeta / 2=\eta-4 \check{\alpha}^{2} / \zeta$. This together with (3.11) implies that

$$
\mathbb{E}_{\mathcal{G}}|x(t)|^{2} \leq\left(\left|x_{0}\right|^{2}+\eta I(0)\right) e^{2 \int_{0}^{t} \phi(s) d s}+\left(2 \check{E}+\frac{\zeta}{4}\right) \int_{0}^{t} e^{2 \int_{s}^{t} \phi(z) d z} d s .
$$

Taking expectation on both sides yields

$$
\mathbb{E}|x(t)|^{2} \leq\left(\left|x_{0}\right|^{2}+\eta I(0)\right) \mathbb{E}\left(e^{2 \int_{0}^{t} \phi(s) d s}\right)+\left(2 \check{E}+\frac{\zeta}{4}\right) \int_{0}^{t} \mathbb{E}\left(e^{2 \int_{s}^{t} \phi(z) d z}\right) d s .
$$

Since $\kappa_{\alpha-D}>2$, by virtue of Lemma 2.6, we have

$$
\mathbb{E}\left(e^{\left.2 \int_{s}^{t}(D(r(z)))-\alpha\left(r\left(\nu(z)-\tau_{0}\right)\right)\right) d z}\right) \leq K_{2}(2, \alpha-D) e^{-\zeta(t-s)}, \quad t \geq s, s \geq 0,
$$

which implies

$$
\mathbb{E}\left(e^{2 \int_{s}^{t} \phi(z) d z}\right) \leq K_{2}(2, \alpha-D) e^{-\zeta(t-s) / 4}, \quad t \geq s, s \geq 0 .
$$

It follows from (3.14) that for any $t \geq 0$,

$$
\int_{0}^{t} \mathbb{E}\left(e^{2 \int_{s}^{t} \phi(z) d z}\right) d s \leq K_{2}(2, \alpha-D) \int_{0}^{t} e^{-\frac{\zeta}{4}(t-s)} d s \leq \frac{4}{\zeta} K_{2}(2, \alpha-D) .
$$

Inserting (3.14) and (3.15) into (3.13) arrives at

$$
\mathbb{E}|x(t)|^{2} \leq\left(\left|x_{0}\right|^{2}+\eta I(0)\right) K_{2}(2, \alpha-D) e^{-\frac{\zeta}{4} t}+\left(\frac{8 \check{E}}{\zeta}+1\right) K_{2}(2, \alpha-D)
$$

for $t \geq 0$. Thus, the required assertion (3.3) follows.

Next we consider the opposite aspect, namely, if the control strength is taken smaller value what will happen. We investigate the longtime behavior of the mean square of the DCSDS (1.3) in this case.

Assumption 3 Assume that there exist positive constants $\bar{K}>0$, and $d_{i}, e_{i}$ such that (3.1) and

$$
x^{T} f(x, i, t)+\frac{1}{2}|g(x, i, t)|^{2} \geq d_{i}|x|^{2}+e_{i}
$$

hold for all $(x, i, t) \in \mathbb{R}^{n} \times \mathbb{S} \times \mathbb{R}_{+}$. 
Theorem 3.2 Let Assumption 3 hold and assume that $v:=\pi d-\pi \alpha>0$, where $d=$ $\left(d_{1}, \cdots, d_{N}\right)^{T}$. If $0<\tau+\tau_{0}<\tau_{*}^{1}=y_{3} \wedge y_{4} \wedge y_{5}$, then the solution of DCSDS (1.3) with the initial solution (2.2) has the property that

$$
\lim _{t \rightarrow \infty} \mathbb{E}|x(t)|^{2}=\infty
$$

where $y_{i}(i=3,4,5)$ are the maximum positive solutions of

$$
\bar{\beta}_{1}(y)=\frac{v(\hat{e} \wedge(v / 2))}{2 \check{\alpha}^{2}+v^{2}}, \quad \bar{\beta}_{3}(y)=\frac{v^{2}}{2 \check{\alpha}^{2}+v^{2}}, \quad \bar{\beta}_{4}(y)=\frac{v\left(\hat{e} /(2 \check{d})+\left|x_{0}\right|^{2}\right)}{2 \check{\alpha}^{2}+v^{2}},
$$

respectively, $\bar{\beta}_{1}(\cdot), \bar{\beta}_{3}(\cdot), \bar{\beta}_{4}(\cdot)$ are defined by (3.6), (3.23), (3.24).

Proof. Fix $0<\tau+\tau_{0} \leq \tau_{*}^{1}$. Using the elementary inequality and (3.16), we derive

$$
\begin{gathered}
d|x(t)|^{2} \geq\left[\left(2 d(r(t))-2 \alpha\left(r\left(\nu(t)-\tau_{0}\right)\right)-v\right)|x(t)|^{2}-\frac{\alpha^{2}}{v}\left|x(t)-x\left(\nu(t)-\tau_{0}\right)\right|^{2}\right. \\
+2 \hat{e}] d t+2 x^{T}(t) g(x(t), r(t), t) d B(t) .
\end{gathered}
$$

Define $U\left(\bar{x}_{t}, \bar{r}_{t}, t\right)=\left(\hat{e} /(2 \check{d})+|x(t)|^{2}-\eta I(t)\right) e^{-2 \int_{0}^{t} \psi(s) d s}$, where $\psi(s):=d(r(s))-\alpha(r(\nu(s)-$ $\left.\left.\tau_{0}\right)\right)-3 v / 4, \eta=v+2 \check{\alpha}^{2} / v, \forall s \geq 0$. One notices that $-(\hat{e} / \check{d}) \psi(t) \geq-\hat{e}$. This together with (3.5) and (3.18) implies that for any $t \geq 0$

$$
\begin{aligned}
d U\left(\bar{x}_{t}, \bar{r}_{t}, t\right) \geq & e^{-2 \int_{0}^{t} \psi(s) d s}\left[\left(2 \eta \psi(t) I(t)+\left(v / 2-\eta \bar{\beta}_{1}\left(\tau+\tau_{0}\right)\right)|x(s)|^{2}\right.\right. \\
+ & \left(\hat{e}-\eta \bar{\beta}_{1}\left(\tau+\tau_{0}\right)\right)-\left(\frac{\check{\alpha}^{2}}{v}+\eta \iota\left(\tau+\tau_{0}\right)\right)\left|x(s)-x\left(\nu(s)-\tau_{0}\right)\right|^{2} \\
& \left.\left.+\eta J_{2}(t)\right) d t+2 x^{T}(t) g(x(t), r(t), t) d B(t)\right] .
\end{aligned}
$$

Due to the increasing property of $\bar{\beta}_{1}(y)$ in $y>0$, one observes

$$
\eta \bar{\beta}_{1}\left(\tau+\tau_{0}\right) \leq(v / 2) \wedge \hat{e} .
$$

It then follows from (2.28) that

$$
2 \eta \psi(t) I(t) \geq-2 \eta(\check{\alpha}+3 v / 4) I(t) \geq-2 \eta(\check{\alpha}+3 v / 4)\left(\tau+\tau_{0}\right) J_{2}(t) .
$$

Inserting (3.20) and (3.21) into (3.19) yields

$$
\begin{aligned}
d U\left(\bar{x}_{t}, \bar{r}_{t}, t\right) & \geq e^{-2 \int_{0}^{t} \psi(s) d s}\left[-\left(\frac{\check{\alpha}^{2}}{v}+\eta \iota\left(\tau+\tau_{0}\right)\right)\left|x(s)-x\left(\nu(s)-\tau_{0}\right)\right|^{2} d t\right. \\
& \left.+\left(\eta-2 \eta\left(\check{\alpha}+\frac{3 v}{4}\right)\left(\tau+\tau_{0}\right)\right) J_{2}(t) d t+2 x^{T}(t) g(x(t), r(t), t) d B(t)\right] .
\end{aligned}
$$

Integrating (3.22) on both sides, taking the conditional expectation with respect to $\mathcal{G}$ and using (2.29), we arrive at

$$
\begin{aligned}
& \mathbb{E}_{\mathcal{G}}\left(e^{-2 \int_{0}^{t} \psi(s) d s}\left(\frac{\hat{e}}{2 \check{d}}+|x(t)|^{2}\right)\right)-\eta \mathbb{E}_{\mathcal{G}}\left(e^{-2 \int_{0}^{t} \psi(s) d s} I(t)\right) \\
& \geq \frac{\hat{e}}{2 \check{d}}+\left|x_{0}\right|^{2}-\eta I(0)+\left(\eta-\frac{2}{v} \check{\alpha}^{2}-\eta \bar{\beta}_{3}\left(\tau+\tau_{0}\right)\right) \int_{0}^{t} e^{-2 \int_{0}^{s} \psi(z) d z} \mathbb{E}_{\mathcal{G}}\left[J_{2}(s)\right] d s,
\end{aligned}
$$


where for $y \geq 0$,

$$
\bar{\beta}_{3}(y):=2 \iota(y)+2 y(\check{\alpha}+3 v / 4)=y\left(8 \check{\alpha}^{2} y+2 \check{\alpha}+3 v / 2\right) .
$$

Then $\eta \bar{\beta}_{3}\left(\tau+\tau_{0}\right) \leq v$. This together with the above inequality implies

$$
\frac{\hat{e}}{2 \check{d}}+\mathbb{E}_{\mathcal{G}}|x(t)|^{2} \geq\left(\frac{\hat{e}}{2 \check{d}}+\left|x_{0}\right|^{2}-\eta I(0)\right) e^{2 \int_{0}^{t} \psi(s) d s} .
$$

Due to (2.27) and (2.28) one observes from (3.1) that $I(0) \leq\left(\tau+\tau_{0}\right) J_{2}(0) \leq \bar{\beta}_{4}\left(\tau+\tau_{0}\right)$, where

$$
\bar{\beta}_{4}(y)=y^{2}\left[3 y\left(\bar{K}^{2}+\check{\alpha}^{2}\right)\left|x_{0}\right|^{2}+2 \bar{K}^{2}\left|x_{0}\right|^{2}+\bar{K}^{2}(3 y+2)\right] .
$$

Taking expectation on both sides yields

$$
\frac{\hat{e}}{2 \check{d}}+\mathbb{E}|x(t)|^{2} \geq\left(\frac{\hat{e}}{2 \check{d}}+\left|x_{0}\right|^{2}-\eta \bar{\beta}_{4}\left(\tau+\tau_{0}\right)\right) \mathbb{E}\left(e^{2 \int_{0}^{t} \psi(s) d s}\right)
$$

It follows from the definition of $\tau_{*}^{1}$ that $\eta \bar{\beta}_{4}\left(\tau+\tau_{0}\right)<\hat{e} / 2 \check{d}+\left|x_{0}\right|^{2}$. By virtue of Lemma 2.5 , for $\epsilon=v / 8$, there is a constant $T>0$ such that

$$
\mathbb{E}\left(e^{\left.2 \int_{0}^{t}(d(r(z)))-\alpha\left(r\left(\nu(z)-\tau_{0}\right)\right)\right) d s}\right) \geq K_{1}(2, \alpha-d) e^{7 v t / 4}, \quad t \geq T,
$$

which implies

$$
\mathbb{E}\left(e^{2 \int_{0}^{t} \psi(z) d z}\right) \geq K_{1}(2, \alpha-d) e^{v t / 4}, \quad t \geq T .
$$

Inserting the above inequality into (3.25), we obtain

$$
\frac{\hat{e}}{2 \check{d}}+\mathbb{E}|x(t)|^{2} \geq K_{1}(2, \alpha-d)\left(\frac{\hat{e}}{2 \check{d}}+\left|x_{0}\right|^{2}-\eta \bar{\beta}_{4}\left(\tau+\tau_{0}\right)\right) e^{v t / 4}, \quad t \geq T .
$$

Then the required assertion (3.17) follows.

\subsection{Stabilization}

This subsection is to discuss the stability and instability of DCSDS (1.3) and gives the corresponding criteria. We replace conditions (3.1) and (3.2) by the following assumption in order for the SDS (1.1) to has the trivial solution $x(t) \equiv 0$.

Assumption 4 There exist positive constants $\bar{K}$ and $D_{i}$ such that

$$
|f(x, i, t)| \vee|g(x, i, t)| \leq \bar{K}|x|
$$

and

$$
x^{T} f(x, i, t)+\frac{1}{2}|g(x, i, t)|^{2} \leq D_{i}|x|^{2}
$$

hold for all $(x, i, t) \in \mathbb{R}^{n} \times \mathbb{S} \times \mathbb{R}_{+}$.

Under Assumption 4 we will design the feedback control for the controlled system (1.3) to be exponentially stable in both mean square and almost surely (a.s.). 
Theorem 3.3 Let Assumption 4 hold and assume that $\pi \alpha>\pi D$ and $\kappa_{\alpha-D}>2$. For any $0<\sigma<\zeta_{2, \alpha-D}^{\tilde{\tau}}$, if $0<\tau \leq \tilde{\tau}$ and $0<\tau+\tau_{0} \leq \tau_{2}^{*}(\sigma):=y_{6}(\sigma) \wedge y_{7}(\sigma)$, then the solution of DCSDS (1.3) with the initial solution (2.2) has the properties that

$$
\limsup _{t \rightarrow \infty} \frac{1}{t} \log \mathbb{E}|x(t)|^{2} \leq-\left(\zeta_{2, \alpha-D}^{\tau}-\sigma\right)
$$

and

$$
\limsup _{t \rightarrow \infty} \frac{1}{t} \log (|x(t)|) \leq-\frac{1}{2}\left(\zeta_{2, \alpha-D}^{\tau}-\sigma\right), \quad \mathbb{P}-\text { a.s. }
$$

where $y_{6}$ and $y_{7}$ are the positive solutions of $\tilde{\beta}_{1}(y)=\frac{\sigma^{2}}{8 \check{\alpha}^{2}+\sigma^{2}}$ and $\tilde{\beta}_{2}(y)=\frac{\sigma^{2}}{8 \check{\alpha}^{2}+\sigma^{2}}$, while $\tilde{\beta}_{1}(\cdot)$ and $\tilde{\beta}_{2}(\cdot)$ are defined by (3.34) and (3.37), respectively.

Proof. For any $0<\sigma<\zeta_{2, \alpha-D}^{\tilde{\tau}}$, let $0<\tau \leq \tilde{\tau}$ and $0<\tau+\tau_{0} \leq \tau_{2}^{*}(\sigma)$. By the Itô formula, the elementary inequality, and (3.29), we have

$$
\begin{gathered}
d|x(t)|^{2} \leq\left[\left(2 D(r(t))-2 \alpha\left(r\left(\nu(t)-\tau_{0}\right)\right)+\frac{\sigma}{2}\right)|x(t)|^{2}+\frac{2 \alpha^{2}}{\sigma}\left|x(t)-x\left(\nu(t)-\tau_{0}\right)\right|^{2}\right] d t \\
+2 x^{T}(t) g(x(t), r(t), t) d B(t) .
\end{gathered}
$$

Using (3.28) we compute $I(t)$ and $J_{1}(t)$ defined by (2.24) and (2.26) to get

$$
d I(t) \leq \tilde{\beta}_{1}\left(\tau+\tau_{0}\right)|x(t)|^{2} d t+\kappa\left(\tau+\tau_{0}\right)\left|x(t)-x\left(\nu(t)-\tau_{0}\right)\right|^{2} d t-J_{2}(t) d t,
$$

where, for $y \geq 0$,

$$
\tilde{\beta}_{1}(y):=y\left[3 y\left(\bar{K}^{2}+\check{\alpha}^{2}\right)+\bar{K}^{2}\right], \quad \kappa(y):=3 \check{\alpha}^{2} y^{2} .
$$

Define $V\left(\bar{x}_{t}, \bar{r}_{t}, t\right)=\left(|x(t)|^{2}+\eta I(t)\right) e^{-2 \int_{0}^{t} \phi(s) d s}$, where $\phi(s):=D(r(s))-\alpha\left(r\left(\nu(s)-\tau_{0}\right)\right)+$ $\sigma / 2, \eta:=\sigma / 2+4 \check{\alpha}^{2} / \sigma$. It follows from (3.32) and (3.33) that for any $t \geq 0$,

$$
\begin{aligned}
d V\left(\bar{x}_{t}, \bar{r}_{t}, t\right) \leq & e^{-2 \int_{0}^{t} \phi(s) d s}\left[\left(-2 \eta \phi(t) I(t)-\left(\frac{\sigma}{2}-\eta \tilde{\beta}_{1}\left(\tau+\tau_{0}\right)\right)|x(s)|^{2}\right.\right. \\
+ & \left(\frac{2 \check{\alpha}^{2}}{\sigma}+\eta \kappa\left(\tau+\tau_{0}\right)\right)\left|x(s)-x\left(\nu(s)-\tau_{0}\right)\right|^{2} \\
& \left.\left.-\eta J_{2}(t)\right) d t+2 x^{T}(t) g(x(t), r(t), t) d B(t)\right]
\end{aligned}
$$

By the definition of $\tau_{2}^{*}(\sigma)$ one observes $\eta \tilde{\beta}_{1}\left(\tau+\tau_{0}\right) \leq \sigma / 2$. Integrating (3.35) on both sides, taking the conditional expectation with respect to the $\sigma$-algebra $\mathcal{G}$ and using (2.28) and (2.29) arrives at

$$
\begin{aligned}
& \mathbb{E}_{\mathcal{G}}\left(e^{-2 \int_{0}^{t} \phi(s) d s}|x(t)|^{2}\right)+\eta \mathbb{E}_{\mathcal{G}}\left(e^{-2 \int_{0}^{t} \phi(s) d s} I(t)\right) \\
& \leq\left|x_{0}\right|^{2}+\eta I(0)-\left(\eta-\frac{4 \check{\alpha}^{2}}{\sigma}-\eta \tilde{\beta}_{2}\left(\tau+\tau_{0}\right)\right) \int_{0}^{t} e^{-2 \int_{0}^{s} \phi(z) d z} \mathbb{E}_{\mathcal{G}}\left[J_{2}(s)\right] d s,
\end{aligned}
$$

where, for $y \geq 0$,

$$
\tilde{\beta}_{2}(y):=2 \kappa(y)+2 y \check{\alpha}=2 \check{\alpha} y(3 \check{\alpha} y+1) .
$$

By the definition of $\tau_{2}^{*}(\sigma)$ one sees $\eta \bar{\beta}_{2}\left(\tau+\tau_{0}\right) \leq \sigma / 2$. This together with (3.36) implies that

$$
\mathbb{E}_{\mathcal{G}}|x(t)|^{2} \leq\left(\left|x_{0}\right|^{2}+\eta I(0)\right) e^{2 \int_{0}^{t} \phi(s) d s} .
$$


Taking expectation on both sides, we get that

$$
\mathbb{E}|x(t)|^{2} \leq\left[\left|x_{0}\right|^{2}+\eta I(0)\right] \mathbb{E}\left(e^{2 \int_{0}^{t} \phi(s) d s}\right) .
$$

But, it follows from Lemma 2.6 that

$$
\mathbb{E}\left(e^{2 \int_{0}^{t} \phi(s) d s}\right) \leq K_{2}(2, \alpha-D) e^{-\left(\zeta_{2, \alpha-D}^{\tilde{\tau}}-\sigma\right) t}, \quad t \geq 0 .
$$

Combing (3.38) and (3.39) yields $\lim \sup _{t \rightarrow \infty} \frac{1}{t} \log \mathbb{E}|x(t)|^{2} \leq-\left(\zeta_{2, \alpha-D}^{\tilde{\tau}}-\sigma\right)$, which implies the required assertion (3.30). In a similar fashion as [11, pp. 128-130, Theorem 4.2] was proved, we can get the other required assertion (3.31).

In order to study the instability we impose the following assumption.

Assumption 5 There exist positive constants $\bar{K}>0$, and $d_{i}$ such that (3.28) and

$$
x^{T} f(x, i, t)+\frac{1}{2}|g(x, i, t)|^{2} \geq d_{i}|x|^{2}
$$

hold for all $(x, i, t) \in \mathbb{R}^{n} \times \mathbb{S} \times \mathbb{R}_{+}$.

Theorem 3.4 Let Assumption 5 hold and assume $\pi \alpha<\pi d$. For any $0<\sigma<\pi d-\pi \alpha$, if $0<\tau+\tau_{0}<\tau_{*}^{2}(\sigma):=y_{8}(\sigma) \wedge y_{9}(\sigma) \wedge y_{10}(\sigma)$, then the solution of DCSDS (1.3) with the initial condition (2.2) has the property that

$$
\liminf _{t \rightarrow \infty} \frac{1}{t} \log \mathbb{E}|x(t)|^{2} \geq 2(\pi d-\pi \alpha-\sigma)
$$

where $y_{i}(\sigma)(i=8,9,10)$ are the positive solutions of $\tilde{\beta}_{1}(y)=\frac{\sigma^{2}}{2 \check{\alpha}^{2}+\sigma^{2}}, \tilde{\beta}_{3}(y)=\frac{\sigma^{2}}{2 \check{\alpha}^{2}+\sigma^{2}}$, $\tilde{\beta}_{4}(y)=\frac{\sigma\left|x_{0}\right|^{2}}{2 \check{\alpha}^{2}+\sigma^{2}}$, respectively, while $\tilde{\beta}_{1}(\cdot), \tilde{\beta}_{3}(\cdot), \tilde{\beta}_{4}(\cdot)$ are defined by $(3.34),(3.44),(3.45)$.

Proof. For any $0<\sigma<\pi d-\pi \alpha$, let $0<\tau+\tau_{0} \leq \tau_{*}^{2}(\sigma)$. Using the elementary inequality and (3.40), one has

$$
\begin{gathered}
d|x(t)|^{2} \geq\left[\left(2 d(r(t))-2 \alpha\left(r\left(\nu(t)-\tau_{0}\right)\right)-\sigma\right)|x(t)|^{2}-\frac{\alpha^{2}}{\sigma}\left|x(t)-x\left(\nu(t)-\tau_{0}\right)\right|^{2}\right] d t \\
+2 x^{T}(t) g(x(t), r(t), t) d B(t) .
\end{gathered}
$$

Define $\bar{U}\left(\bar{x}_{t}, \bar{r}_{t}, t\right)=\left(|x(t)|^{2}-\eta I(t)\right) e^{-2 \int_{0}^{t} \psi(s) d s}$, where $\psi(s):=d(r(s))-\alpha\left(r\left(\nu(s)-\tau_{0}\right)\right)-\sigma$, and $\eta:=\sigma+2 \check{\alpha}^{2} / \sigma$. In a similar way as Theorem 3.2 was proved we can obtain from (3.33) and (3.42) that for any $t \geq 0$

$$
\begin{aligned}
d \bar{U}\left(\bar{x}_{t}, \bar{r}_{t}, t\right) \geq & e^{-2 \int_{0}^{t} \psi(s) d s}\left[\left(2 \eta \psi(t) I(t)+\left(\sigma-\eta \tilde{\beta}_{1}\left(\tau+\tau_{0}\right)\right)|x(t)|^{2}\right.\right. \\
& -\left(\frac{\check{\alpha}^{2}}{\sigma}+\eta \kappa\left(\tau+\tau_{0}\right)\right)\left|x(t)-x\left(\nu(t)-\tau_{0}\right)\right|^{2} \\
& \left.\left.+\eta J_{2}(t)\right) d t+2 x^{T}(t) g(x(t), r(t), t) d B(t)\right] .
\end{aligned}
$$


One notices that $\eta \tilde{\beta}_{1}\left(\tau+\tau_{0}\right) \leq \sigma$. Integrating (3.43) on both sides, taking the conditional expectation with respect to the $\sigma$-algebra $\mathcal{G}$ and using (2.28) and (2.29), we arrive at

$$
\begin{aligned}
& \mathbb{E}_{\mathcal{G}}\left(e^{-2 \int_{0}^{t} \psi(s) d s}|x(t)|^{2}\right)-\eta \mathbb{E}_{\mathcal{G}}\left(e^{-2 \int_{0}^{t} \psi(s) d s} I(t)\right) \\
& \geq\left|x_{0}\right|^{2}-\eta I(0)+\left(\eta-\frac{2}{\sigma} \check{\alpha}^{2}-\eta \tilde{\beta}_{3}\left(\tau+\tau_{0}\right)\right) \int_{0}^{t} e^{-2 \int_{0}^{s} \psi(z) d z} \mathbb{E}_{\mathcal{G}}\left[J_{2}(s)\right] d s
\end{aligned}
$$

where, for $y \geq 0$,

$$
\tilde{\beta}_{3}(y):=2 \kappa(y)+2 y(\check{\alpha}+\sigma)=2 y\left(3 \check{\alpha}^{2} y+\check{\alpha}+\sigma\right) .
$$

We also see that $\eta \tilde{\beta}_{3}\left(\tau+\tau_{0}\right) \leq \sigma$. This together with the above inequality implies

$$
\mathbb{E}_{\mathcal{G}}|x(t)|^{2} \geq\left(\left|x_{0}\right|^{2}-\eta I(0)\right) e^{2 \int_{0}^{t} \psi(s) d s} .
$$

Due to (2.27) and (2.28) one observes that $I(0) \leq\left(\tau+\tau_{0}\right) J_{2}(0) \leq \tilde{\beta}_{4}\left(\tau+\tau_{0}\right)$, where

$$
\tilde{\beta}_{4}(y)=y^{2}\left[2 y\left(\bar{K}^{2}+\check{\alpha}^{2}\right)\left|x_{0}\right|^{2}+\bar{K}^{2}\left|x_{0}\right|^{2}\right] .
$$

One notices that $\eta \tilde{\beta}_{4}\left(\tau+\tau_{0}\right)<\left|x_{0}\right|^{2}$. Taking expectation on both sides yields

$$
\mathbb{E}|x(t)|^{2} \geq\left(\left|x_{0}\right|^{2}-\eta \tilde{\beta}_{4}\left(\tau+\tau_{0}\right)\right) \mathbb{E}\left(e^{2 \int_{0}^{t} \psi(s) d s}\right) .
$$

By Lemma 2.5, for $0<\epsilon<\pi d-\pi \alpha-\sigma$, there is a constant $T>0$ such that

$$
\mathbb{E}|x(t)|^{2} \geq\left(\left|x_{0}\right|^{2}-\eta \tilde{\beta}_{4}\left(\tau+\tau_{0}\right)\right) e^{2(\pi d-\pi \alpha-\sigma-\epsilon) t}, \quad t \geq T .
$$

Letting $t \rightarrow \infty$, we have $\liminf _{t \rightarrow \infty} \frac{1}{t} \log \mathbb{E}|x(t)|^{2} \geq 2(\pi d-\pi \alpha-\sigma-\epsilon)$. As $\epsilon>0$ is arbitrary, the required assertion (3.41) must hold.

\section{Control of Highly Nonlinear Systems}

The main aim of this section is to give the easily implementable control criterion for highly nonlinear SDS (1.1) such that they stabilize (1.1) exponentially in $p$ th moment and almost surely. In the following, the moment and sample Lyapunov exponents are estimated, the lower bound on $\tau+\tau_{0}$ is given explicitly.

\subsection{Uniform Moment Boundness}

Firstly we investigate the uniform moment boundedness of DCSDS (1.3). Generally, SFDEs have significantly different dynamical behaviors from the corresponding SDSs. Hence the uncontrolled SDS (1.1) may possess some property while the DCSDS (1.3) may not. We impose the following Khasminskii-type condition to guarantee that the global solution of the SDS (1.1) is uniformly bounded in $p$ th moment on infinite time horizon. 
Assumption 6 There exist positive constants $A, B, C$ and $p \geq 2, \theta>2$ such that

$$
x^{T} f(x, i, t)+\frac{p-1}{2}|g(x, i, t)|^{2} \leq C+A|x|^{2}-B|x|^{\theta}
$$

for all $(x, i, t) \in \mathbb{R}^{n} \times \mathbb{S} \times \mathbb{R}_{+}$.

By constructing $V(x, i, t)=|x|^{p}$ for all $(x, i, t) \in \mathbb{R}^{n} \times \mathbb{S} \times \mathbb{R}_{+}$, and using [15, p. 157, Theorem 5.2] we can get the following result directly. To avoid the duplication we omit the proof details.

Theorem 4.1 Under Assumption 6, the solution $x(t)$ of $S D S$ (1.1) with the initial data $(x(0), r(0))=\left(x_{0}, i_{0}\right) \in \mathbb{R}^{n} \times \mathbb{S}$ satisfies $\sup _{0 \leq t<\infty} \mathbb{E}|x(t)|^{p}<\infty$.

For the DCSDS (1.3) we have the following result.

Theorem 4.2 Under Assumption 6, the solution $x(t)$ of DCSDS (1.3) with the initial data (2.2) satisfies $\sup _{0 \leq t<\infty} \mathbb{E}|x(t)|^{p}<\infty$.

Proof. Using the Itô formula and Assumption 6, we derives that, for any $t \geq 0$,

$$
\begin{aligned}
d\left(e^{t}|x(t)|^{p}\right) \leq e^{t} & {\left[p C|x(t)|^{p-2}+(1+p A)|x(t)|^{p}-p B|x(t)|^{p+\theta-2}\right] d t } \\
& +\check{\alpha} p e^{t}|x(t)|^{p-1}\left|x\left(\nu(t)-\tau_{0}\right)\right| d t \\
& +p e^{t}|x(t)|^{p-2} x^{T}(t) g(x(t), r(t), t) d B(t) .
\end{aligned}
$$

Noting that for any $x, y \geq 0$

$$
x^{p-2} \leq 1+x^{p}, \quad \check{\alpha} p x^{p-1} y \leq \frac{p-1}{p}(\check{\alpha} p)^{\frac{p}{p-1}} x^{p}+\frac{1}{p} y^{p}=(p-1) p^{\frac{1}{p-1}}(\check{\alpha})^{\frac{p}{p-1}} x^{p}+\frac{1}{p} y^{p},
$$

we have

$$
\begin{aligned}
& d\left(e^{t}|x(t)|^{p}\right) \\
\leq & e^{t}\left(\bar{C}+\frac{1}{p}\left|x\left(\nu(t)-\tau_{0}\right)\right|^{p}\right) d t+p e^{t}|x(t)|^{p-2} x^{T}(t) g(x(t), r(t), t) d B(t),
\end{aligned}
$$

where

$$
\bar{C}:=\sup _{x \in \mathbb{R}_{+}}\left\{p C+\left(1+p A+p C+(p-1) p^{\frac{1}{p-1} \check{\alpha}^{\frac{p}{p-1}}}\right) x^{p}-p B x^{p+\theta-2}\right\}
$$

Integrating (4.1) from 0 to $t$, taking expectations, then dividing $e^{t}$ on both sides, we obtain

$$
\begin{aligned}
\mathbb{E}|x(t)|^{p} & \leq \bar{C}+\left|x_{0}\right|^{p} e^{-t}+\frac{1}{p} \int_{0}^{t} e^{s-t} \mathbb{E}\left|x\left(\nu(s)-\tau_{0}\right)\right|^{p} d s \\
& \leq \bar{C}+\left|x_{0}\right|^{p}+\frac{1}{p} \sup _{0 \leq s \leq t}\left(\mathbb{E}\left|x\left(\nu(s)-\tau_{0}\right)\right|^{p}\right) \int_{0}^{t} e^{s-t} d s \\
& \leq \bar{C}+\left|x_{0}\right|^{p}+\frac{1}{p} \sup _{0 \leq s \leq t}\left(\mathbb{E}|x(s)|^{p}\right) .
\end{aligned}
$$

This implies $\sup _{0 \leq s \leq t}\left(\mathbb{E}|x(s)|^{p}\right) \leq \frac{p\left(\bar{C}+\left|x_{0}\right|^{p}\right)}{p-1}$. Then the required assertion follows as $t \rightarrow \infty$. 
Since the coefficients of SDSs (1.1) depend on the Markov chain modes, Theorem 4.2 can be extended to more general results as follows.

Corollary 4.3 Assume that there exist positive constants $p \geq 2$ and $A_{i}, B_{i}, C_{i}, \theta_{i}>2$ such that

$$
x^{T} f(x, i, t)+\frac{p-1}{2}|g(x, i, t)|^{2} \leq C_{i}+A_{i}|x|^{2}-B_{i}|x|^{\theta_{i}}
$$

holds for all $(x, i, t) \in \mathbb{R}^{n} \times \mathbb{S} \times \mathbb{R}_{+}$. Then the results of Theorem 4.2 still hold.

Proof. For each $i \in \mathbb{S},|x|^{\hat{\theta}} \leq 1+|x|^{\theta_{i}}$. This implies $-|x|^{\theta_{i}} \leq 1-|x|^{\hat{\theta}}$. Then the right side of (4.3) satisfies

$$
C_{i}+A_{i}|x|^{2}-B_{i}|x|^{\theta_{i}} \leq \check{C}+\check{A}|x|^{2}-\hat{B}|x|^{\theta_{i}} \leq(\check{C}+\hat{B})+\check{A}|x|^{2}-\hat{B}|x|^{\hat{\theta}} .
$$

Thus (4.3) implies that Assumption 6 holds. So the required results follows.

\subsection{Stabilization}

In this subsection we pay attention to stabilize the nonlinear SDS (1.1) by the delay feedback control based on discrete-time observations. In order to have the equilibrium state 0 we further impose the following assumption.

Assumption 7 Assume that there exist positive constants $K, q_{1} \geq 1, q_{2} \geq 1, p \geq$ $2\left(q_{1} \vee q_{2}\right), \theta>2$ satisfying $\theta \geq\left(q_{1} \vee q_{2}\right)+1$, and $A_{i}, B_{i}$ such that

$$
|f(x, i, t)| \leq K\left(|x|+|x|^{q_{1}}\right), \quad|g(x, i, t)| \leq K\left(|x|+|x|^{q_{2}}\right)
$$

and

$$
x^{T} f(x, i, t)+\frac{p-1}{2}|g(x, i, t)|^{2} \leq A_{i}|x|^{2}-B_{i}|x|^{\theta}
$$

hold for all $(x, i, t) \in \mathbb{R}^{n} \times \mathbb{S} \times \mathbb{R}_{+}$.

Theorem 4.4 Let Assumption 7 hold and assume that $\pi \alpha>\pi A$ and $\kappa_{\alpha-A}>2$, where $A=\left(A_{1}, \cdots, A_{N}\right)^{T}$. For any $0<\sigma<\zeta_{2, \alpha-A}^{\tau^{\prime}} \wedge(2 \hat{B})\left(\tau^{\prime}:=\bar{\tau}(2, \alpha-A) / 2\right)$, if $0<\tau \leq \tau^{\prime}$ and $0<\tau+\tau_{0}<\tau^{* *}(\sigma):=\bar{y}_{1}(\sigma) \wedge \bar{y}_{2}(\sigma) \wedge \bar{y}_{3}(\sigma)$, then the solution of DCSDS (1.3) with the initial condition (2.2) has the properties that

$$
\begin{aligned}
& \limsup _{t \rightarrow \infty} \frac{1}{t} \log \mathbb{E}|x(t)|^{2} \leq-\left(\zeta_{2, \alpha-A}^{\tau}-\sigma\right), \\
& \limsup _{t \rightarrow \infty} \frac{1}{t} \log \mathbb{E}|x(t)|^{\rho} \leq-\left(\zeta_{2, \alpha-A}^{\tau}-\sigma\right),
\end{aligned}
$$

and

$$
\int_{0}^{\infty} \mathbb{E}|x(t)|^{\rho+\theta-2} d t<\infty,
$$

where $\rho:=p \wedge \theta, \bar{y}_{i}(\sigma)(i=1,2,3)$ are the positive solutions of

$$
2 \vartheta \beta_{1}(y)=\sigma, \quad 2 \vartheta \beta_{2}(y)=\rho(2 \hat{B}-\sigma), \quad 2 \vartheta \beta_{3}(y)=\sigma,
$$

respectively, $\vartheta:=\sigma / 2+\check{\alpha}^{2}[(5 \rho+4) \sigma+8(\rho-2) \check{A}] /\left(\sigma^{2}\right), \beta_{1}(\cdot), \beta_{2}(\cdot), \beta_{3}(\cdot)$ are defined by (4.12) and (4.23). 
Proof. For any $0<\sigma<\zeta_{2, \alpha-A}^{\tau^{\prime}} \wedge(2 \hat{B})$, let $0<\tau \leq \tau^{\prime}$ and $0<\tau+\tau_{0} \leq \tau^{* *}(\sigma)$. Using the Itô formula, the elementary inequality and (4.5), we derive

$$
\begin{aligned}
& d|x(t)|^{\rho} \\
& \leq\left[\rho\left(A(r(t))-\alpha\left(r\left(\nu(t)-\tau_{0}\right)\right)\right)|x(t)|^{\rho}-\rho\left(\hat{B}-\frac{\sigma}{2}\right)|x(t)|^{\rho+\theta-2}+\frac{\rho \sigma}{2}|x(t)|^{2}\right. \\
& \left.\quad+\frac{\rho \check{\alpha}^{2}}{2 \sigma}\left|x(t)-x\left(\nu(t)-\tau_{0}\right)\right|^{2}\right] d t+\rho|x(t)|^{\rho-2} x^{T}(t) g(x(t), r(t), t) d B(t) .
\end{aligned}
$$

In order to control the terms $|x(t)|^{\rho}$ and $|x(t)|^{2}$ together, we also derive by the Itô formula again that

$$
\begin{aligned}
d|x(t)|^{2} \leq & 2\left(A(r(t))-\alpha\left(r\left(\nu(t)-\tau_{0}\right)\right)+\frac{\sigma}{4}\right)|x(t)|^{2} d t-2 \hat{B}|x(t)|^{\theta} d t \\
& +\frac{2 \check{\alpha}^{2}}{\sigma}\left|x(t)-x\left(\nu(t)-\tau_{0}\right)\right|^{2} d t+2 x^{T}(t) g(x(t), r(t), t) d B(t) .
\end{aligned}
$$

Under the condition (4.4) we recompute $I(t)$ and $J_{1}(t)$ defined by $(2.24)$ and $(2.26)$

$$
\begin{aligned}
J_{1}(t) \leq & \left(\tau+\tau_{0}\right)\left[\left(3\left(\tau+\tau_{0}\right)\left(K^{2}+2 \check{\alpha}^{2}\right)+2 K^{2}\right)|x(t)|^{2}\right. \\
& \left.+6 \check{\alpha}^{2}\left(\tau+\tau_{0}\right)\left|x(t)-x\left(\nu(t)-\tau_{0}\right)\right|^{2}+3 K^{2}\left(\tau+\tau_{0}\right)|x(t)|^{2 q_{1}}+2 K^{2}|x(t)|^{2 q_{2}}\right] \\
\leq & \left(\tau+\tau_{0}\right)\left[\left(6\left(\tau+\tau_{0}\right)\left(K^{2}+\check{\alpha}^{2}\right)+4 K^{2}\right)|x(t)|^{2}\right. \\
& \left.+K^{2}\left(3\left(\tau+\tau_{0}\right)+2\right)|x(t)|^{\rho+\theta-2}+6 \check{\alpha}^{2}\left(\tau+\tau_{0}\right)\left|x(t)-x\left(\nu(t)-\tau_{0}\right)\right|^{2}\right],
\end{aligned}
$$

where we have used $\rho+\theta-2 \geq 2\left(q_{1} \vee q_{2}\right)$. By (2.25) one has

$$
\begin{aligned}
d I(t) \leq \beta_{1}(\tau+ & \left.\tau_{0}\right)|x(t)|^{2} d t+\beta_{2}\left(\tau+\tau_{0}\right)|x(t)|^{\rho+\theta-2} d t \\
& +6 \check{\alpha}^{2}\left(\tau+\tau_{0}\right)^{2}\left|x(t)-x\left(\nu(t)-\tau_{0}\right)\right|^{2} d t-J_{2}(t) d t
\end{aligned}
$$

where, for $y \geq 0$,

$$
\beta_{1}(y):=2 y\left[3 y\left(K^{2}+\check{\alpha}^{2}\right)+2 K^{2}\right], \quad \beta_{2}(y):=K^{2} y[3 y+2] .
$$

Define $\bar{V}\left(\bar{x}_{t}, \bar{r}_{t}, t\right)=|x(t)|^{\rho}+\lambda|x(t)|^{2}+\vartheta I(t)$, where $\lambda:=1+\rho+2(\rho-2) \check{A} / \sigma$, and $\vartheta$ is given in the theorem. For any $t \geq 0$, define $\varphi(t):=A(r(t))-\alpha\left(r\left(\nu(t)-\tau_{0}\right)\right)+\sigma / 2$. Using (4.8), (4.9) and (4.11) arrives at

$$
\begin{aligned}
& d \bar{V}\left(\bar{x}_{t}, \bar{r}_{t}, t\right) \\
& \leq\left[\rho\left(A(r(t))-\alpha\left(r\left(\nu(t)-\tau_{0}\right)\right)\right)|x(t)|^{\rho}-\left(\rho \hat{B}-\frac{\rho \sigma}{2}-\vartheta \beta_{2}\left(\tau+\tau_{0}\right)\right)|x(t)|^{\rho+\theta-2}\right. \\
& \quad-2 \lambda \hat{B}|x(t)|^{\theta}+\left(2 \lambda \varphi(t)+\frac{\rho \sigma}{2}-\frac{\lambda \sigma}{2}+\vartheta \beta_{1}\left(\tau+\tau_{0}\right)\right)|x(t)|^{2}-\vartheta J_{2}(t) \\
& \left.\quad+\left(\frac{(\rho+4 \lambda) \check{\alpha}^{2}}{2 \sigma}+6 \vartheta \check{\alpha}^{2}\left(\tau+\tau_{0}\right)^{2}\right)\left|x(t)-x\left(\nu(t)-\tau_{0}\right)\right|^{2}\right] d t \\
& \quad+\left(\rho|x(t)|^{\rho-2}+2 \lambda\right) x^{T}(t) g(x(t), r(t), t) d B(t) .
\end{aligned}
$$

Since $\varphi(s)$ has a finite number of jumps in any finite interval $[0, t], \int_{0}^{t} \varphi(s) d s$ is derivable. 
Thus, it follows from (4.13) that

$$
\begin{aligned}
& d\left(e^{-2 \int_{0}^{t} \varphi(s) d s} \bar{V}\left(\bar{x}_{t}, \bar{r}_{t}, t\right)\right) \\
&=e^{-2 \int_{0}^{t} \varphi(s) d s}\left(-2 \varphi(t) \bar{V}\left(\bar{x}_{t}, \bar{r}_{t}, t\right) d t+d \bar{V}\left(\bar{x}_{t}, \bar{r}_{t}, t\right)\right) \\
&=e^{-2 \int_{0}^{t} \varphi(s) d s[}-2 \vartheta \varphi(t) I(t)+(\rho-2)\left(A(r(t))-\alpha\left(r\left(\nu(t)-\tau_{0}\right)\right)\right)|x(t)|^{\rho} \\
&-\sigma|x(t)|^{\rho}-\left(\rho \hat{B}-\frac{\rho \sigma}{2}-\vartheta \beta_{2}\left(\tau+\tau_{0}\right)\right)|x(t)|^{\rho+\theta-2} \\
&-2 \lambda \hat{B}|x(t)|^{\theta}-\left(\frac{(\lambda-\rho) \sigma}{2}-\vartheta \beta_{1}\left(\tau+\tau_{0}\right)\right)|x(t)|^{2}-\vartheta J_{2}(t) \\
&\left.+\left(\frac{(\rho+4 \lambda) \check{\alpha}^{2}}{2 \sigma}+6 \vartheta \check{\alpha}^{2}\left(\tau+\tau_{0}\right)^{2}\right)\left|x(t)-x\left(\nu(t)-\tau_{0}\right)\right|^{2}\right] d t \\
&+e^{-2 \int_{0}^{t} \varphi(s) d s}\left(\rho|x(t)|^{\rho-2}+2 \lambda\right) x^{T}(t) g(x(t), r(t), t) d B(t) .
\end{aligned}
$$

One observes from (2.28) that

$$
-2 \vartheta \varphi(t) I(t) \leq 2 \vartheta \check{\alpha} I(t) \leq 2 \vartheta \check{\alpha}\left(\tau+\tau_{0}\right) J_{2}(t) .
$$

Noticing $2 \leq \rho \leq \theta$, we obtain

$$
\left(A(r(t))-\alpha\left(r\left(\nu(t)-\tau_{0}\right)\right)\right)|x(t)|^{\rho} \leq \check{A}|x(t)|^{\rho} \leq \check{A}|x(t)|^{2}+\check{A}|x(t)|^{\theta} .
$$

Inserting (4.15) and (4.16) into (4.14) yields

$$
\begin{gathered}
d\left(e^{-2 \int_{0}^{t} \varphi(s) d s} \bar{V}\left(\bar{x}_{t}, \bar{r}_{t}, t\right)\right) \\
\leq e^{-2 \int_{0}^{t} \varphi(s) d s}\left[-\left(\rho \hat{B}-\frac{\rho \sigma}{2}-\vartheta \beta_{2}\left(\tau+\tau_{0}\right)\right)|x(t)|^{\rho+\theta-2}-(2 \lambda \hat{B}-(\rho-2) \check{A})|x(t)|^{\theta}\right. \\
-\left(\frac{(\lambda-\rho) \sigma}{2}-(\rho-2) \check{A}-\vartheta \beta_{1}\left(\tau+\tau_{0}\right)\right)|x(t)|^{2}-\vartheta\left(1-2 \check{\alpha}\left(\tau+\tau_{0}\right)\right) J_{2}(t) \\
\left.+\left(\frac{(\rho+4 \lambda) \check{\alpha}^{2}}{2 \sigma}+6 \vartheta \check{\alpha}^{2}\left(\tau+\tau_{0}\right)^{2}\right)\left|x(t)-x\left(\nu(t)-\tau_{0}\right)\right|^{2}\right] d t \\
+e^{-2 \int_{0}^{t} \varphi(s) d s}\left(\rho|x(t)|^{\rho-2}+2 \lambda\right) x^{T}(t) g(x(t), r(t), t) d B(t) .
\end{gathered}
$$

One notices from $0<\sigma<2 \hat{B}$ and the definition of $\lambda$ that

$$
2 \lambda \hat{B}-(\rho-2) \check{A}>0 .
$$

From the definitions of $\tau^{* *}(\sigma), \lambda, \beta_{1}(\cdot)$ and $\beta_{2}(\cdot)$, one notices that $\tau^{* *}(\sigma)<1$, and furthermore

$$
\vartheta \beta_{1}\left(\tau+\tau_{0}\right) \leq \frac{\sigma}{2}=\frac{\sigma(\lambda-\rho)}{2}-(\rho-2) \check{A}, \quad \vartheta \beta_{2}\left(\tau+\tau_{0}\right) \leq \rho\left(\hat{B}-\frac{\sigma}{2}\right) .
$$

Inserting (4.18)-(4.19) into (4.17) yields

$$
\begin{aligned}
& d\left(e^{-2 \int_{0}^{t} \varphi(s) d s} \bar{V}\left(\bar{x}_{t}, \bar{r}_{t}, t\right)\right) \\
& \leq e^{-2 \int_{0}^{t} \varphi(s) d s}\left[\left(\frac{(\rho+4 \lambda) \check{\alpha}^{2}}{2 \sigma}+6 \vartheta \check{\alpha}^{2}\left(\tau+\tau_{0}\right)^{2}\right)\left|x(t)-x\left(\nu(t)-\tau_{0}\right)\right|^{2} d t\right. \\
& \left.\quad-\vartheta\left(1-2 \check{\alpha}\left(\tau+\tau_{0}\right)\right) J_{2}(t) d t+\left(\rho|x(t)|^{\rho-2}+2 \lambda\right) x^{T}(t) g(x(t), r(t), t) d B(t)\right] .
\end{aligned}
$$


Using (2.28) implies that

$$
\bar{V}(\bar{x}(0), \bar{r}(0), 0) \leq\left|x_{0}\right|^{\rho}+\lambda\left|x_{0}\right|^{2}+\vartheta\left(\tau+\tau_{0}\right) J_{2}(0)<\infty .
$$

Integrating (4.20) on both sides, taking the conditional expectation with respect to the $\sigma$-algebra $\mathcal{G}$ and using (4.21), (2.29), we obtain

$$
\begin{aligned}
& e^{-2 \int_{0}^{t} \varphi(s) d s} \mathbb{E}_{\mathcal{G}} \bar{V}\left(\bar{x}_{t}, \bar{r}_{t}, t\right) \leq \bar{V}(\bar{x}(0), \bar{r}(0), 0) \\
& \quad-\int_{0}^{t} e^{-2 \int_{0}^{s} \varphi(z) d z}\left[\left(\vartheta-\frac{(\rho+4 \lambda) \check{\alpha}^{2}}{\sigma}-\vartheta \beta_{3}\left(\tau+\tau_{0}\right)\right) \mathbb{E}_{\mathcal{G}}\left[J_{2}(s)\right]\right] d s,
\end{aligned}
$$

where for any $y \geq 0$,

$$
\beta_{3}(y):=2 \check{\alpha} y(1+6 \check{\alpha} y) .
$$

Due to $p \geq 2\left(q_{1} \vee q_{2}\right)$ one observes from Theorem 4.2 that $\mathbb{E}_{\mathcal{G}}\left[J_{2}(s)\right]<\infty$ for any $s \geq 0$. It follows from the definitions of $\tau^{* *}(\sigma), \lambda, \vartheta$ that $\vartheta \beta_{3}\left(\tau+\tau_{0}\right) \leq \frac{\sigma}{2}=\vartheta-\frac{(\rho+4 \lambda) \check{\alpha}^{2}}{\sigma}$. This together with (4.22) implies

$$
\mathbb{E}_{\mathcal{G}} \bar{V}\left(\bar{x}_{t}, \bar{r}_{t}, t\right) \leq \bar{V}(\bar{x}(0), \bar{r}(0), 0) e^{2 \int_{0}^{t} \varphi(s) d s} d s
$$

Then

$$
\mathbb{E}|x(t)|^{\rho}+\lambda \mathbb{E}|x(t)|^{2} \leq \bar{V}(\bar{x}(0), \bar{r}(0), 0) \mathbb{E}\left(e^{2 \int_{0}^{t} \varphi(s) d s}\right) .
$$

It follows from Lemma 2.6 that

$$
\mathbb{E}|x(t)|^{\rho}+\lambda \mathbb{E}|x(t)|^{2} \leq \bar{V}(\bar{x}(0), \bar{r}(0), 0) e^{-\left(\zeta_{2, \alpha-A}^{\tau}-\sigma\right) t},
$$

which implies that (4.6) holds. Integrating (4.13) on both sides, taking expectation, and using the similar techniques yields

$$
\begin{aligned}
& \left(\rho \hat{B}-\frac{\rho \sigma}{2}-\vartheta \beta_{2}\left(\tau+\tau_{0}\right)\right) \int_{0}^{t} \mathbb{E}|x(s)|^{\rho+\theta-2} d s \\
& \leq \bar{V}(\bar{x}(0), \bar{r}(0), 0)+\rho\left(\check{A}+\frac{\sigma}{2}\right) \int_{0}^{t}\left(\mathbb{E}|x(s)|^{\rho}+\lambda \mathbb{E}|x(s)|^{2}\right) d s .
\end{aligned}
$$

This, together with (4.24), implies

$$
\left(\rho \hat{B}-\frac{\rho \sigma}{2}-\vartheta \beta_{2}\left(\tau+\tau_{0}\right)\right) \int_{0}^{t} \mathbb{E}|x(s)|^{\rho+\theta-2} d s \leq \bar{C}_{1},
$$

where $\bar{C}_{1}$ is a positive constant. The conclusion (4.7) follows by letting $t \rightarrow \infty$.

The corresponding results for a special case $p \geq \theta$ follows directly from the above proof but holds for a possible bigger $\tau^{* *}(\sigma)$.

Corollary 4.5 Let Assumption 7 hold with $p \geq \theta, \pi \alpha>\pi A$ and $\kappa_{\alpha-A}>2$. For any $0<\sigma<\zeta_{2, \alpha-A}^{\tau^{\prime}} \wedge(2 \hat{B})$, if $0<\tau \leq \tau^{\prime}$ and $0<\tau+\tau_{0}<\bar{\tau}^{* *}(\sigma):=\bar{y}_{1}^{\prime}(\sigma) \wedge \bar{y}_{2}^{\prime}(\sigma) \wedge \bar{y}_{3}^{\prime}(\sigma)$, the conclusions of Theorem 4.4 hold with $\rho=\theta$, where $\bar{y}_{i}^{\prime}(\sigma)(i=1,2,3)$ are the positive solutions of $4 \hat{B} \vartheta_{1} \beta_{1}(y)=\sigma(\theta-2) \check{A}, 2 \vartheta_{1} \beta_{2}(y)=\theta(2 \hat{B}-\sigma), 2 \vartheta_{1} \beta_{3}(y)=\sigma$, respectively, $\vartheta_{1}:=\sigma / 2+\check{\alpha}^{2}[\theta \hat{B}+2(\theta-2) \check{A}] /(\sigma \hat{B}), \beta_{1}(\cdot), \beta_{2}(\cdot), \beta_{3}(\cdot)$ are defined by (4.12) and (4.23). 
Due to the uniform boundedness of $\mathbb{E}|x(t)|^{p}$ on the infinite horizon, by the Hölder inequality, we go one step further to obtain the following result.

Theorem 4.6 Under the conditions of Theorem 4.4, for any $0<\sigma<\zeta_{2, \alpha-A}^{\tau^{\prime}} \wedge(2 \hat{B})$, if $0<\tau \leq \tau^{\prime}$ and $0<\tau+\tau_{0}<\bar{\tau}^{* *}(\sigma)$, the solution of DCSDS (1.3) with the initial condition (2.2) has the property that for any $q \in[2, p)$

$$
\limsup _{t \rightarrow \infty} \frac{1}{t} \log \left(\mathbb{E}|x(t)|^{q}\right) \leq \xi_{q}:= \begin{cases}-\left(\zeta_{2, \alpha-A}^{\tau}-\sigma\right), & q=2, \\ -\frac{q}{\rho}\left(\zeta_{2, \alpha-A}^{\tau}-\sigma\right), & q \in(2, \rho], \\ -\frac{p-q}{p-\rho}\left(\zeta_{2, \alpha-A}^{\tau}-\sigma\right), & q \in(\rho, p), \quad(\text { if } \rho<p)\end{cases}
$$

Using the techniques of $[8$, p.10, Theorem 4.5] we can obtain the following sample Lyapunov exponent. But to avoid duplication we omit the proof.

Theorem 4.7 Under the conditions of Theorem 4.4 and $p>v:=\left(2 q_{1}\right) \vee\left(2 q_{2}\right)$, for any $0<\sigma<\zeta_{2, \alpha-A}^{\tau^{\prime}} \wedge(2 \hat{B})$, if $0<\tau \leq \tau^{\prime}$ and $0<\tau+\tau_{0} \leq \tau^{* *}(\sigma)$, the solution of DCSDS (1.3) with the initial solution (2.2) has the property that

$$
\limsup _{t \rightarrow \infty} \frac{1}{t} \log (|x(t)|) \leq-\xi_{v} \quad \text { a.s. }
$$

where the definition of $\xi$. is given by (4.25). This implies that the DCSDS (1.3) is almost surely exponentially stable

Since the coefficients of the SDS (1.1) depend on the Markov chain modes, Theorems 4.4, 4.6 and 4.7 can be extended to more general results as follows.

Assumption 8 Assume that there exist positive constants $K, q_{1} \geq 1, q_{2} \geq 1, p \geq$ $2\left(q_{1} \vee q_{2}\right)$ and $A_{i}, B_{i}, \theta_{i}>2$ satisfying $\hat{\theta} \geq q_{1} \vee q_{2}+1$ such that (4.4) and

$$
x^{T} f(x, i, t)+\frac{p-1}{2}|g(x, i, t)|^{2} \leq A_{i}|x|^{2}-B_{i}|x|^{\theta_{i}}
$$

hold for all $(x, i, t) \in \mathbb{R}^{n} \times \mathbb{S} \times \mathbb{R}_{+}$.

For each $i \in \mathbb{S},|x|^{\hat{\theta}} \leq|x|^{2}+|x|^{\theta_{i}}$, which implies $-|x|^{\theta_{i}} \leq|x|^{2}-|x|^{\hat{\theta}}$. Then the right side of (4.27) satisfies

$$
A_{i}|x|^{2}-B_{i}|x|^{\theta_{i}} \leq\left(A_{i}+B_{i}\right)|x|^{2}-B_{i}|x|^{\hat{\theta}}
$$

Thus Assumption 8 implies that Assumption 4 holds. So we have the following corollary.

Corollary 4.8 Let Assumption 8, $\pi \alpha>\pi A+\pi B$ and $\kappa_{\alpha-A}>2$ hold. Then the corresponding conclusions of Theorems 4.4, 4.6 and 4.7 still hold. 


\section{Example}

Example 5.1 Consider a scalar nonlinear SDS (1.1) with a scalar Brownian motion $B(t)$, a Markov chain $r(t)$ on the state space $\mathbb{S}=\{1,2\}$ with its generator matrix $\Gamma=$ $\left(\begin{array}{cc}-10 & 10 \\ 20 & -20\end{array}\right)$, and the coefficients $f$ and $g$ defined by

$$
\begin{array}{ll}
f(x, 1, t)=x\left(1-3 x^{2}\right) d t, & g(x, 1, t)=|x|^{3 / 2} \\
f(x, 2, t)=x\left(1-2 x^{2}\right) d t, & g(x, 2, t)=x .
\end{array}
$$

One observes that (4.4) is satisfied with $q_{1}=3, q_{2}=3 / 2, K=3$. Due to the Young inequality one goes a further step to obtain that

$$
\begin{aligned}
& x^{T} f(x, 1, t)+\frac{7-1}{2}|g(x, 1, t)|^{2}=|x|^{2}+3|x|^{3}-3|x|^{4} \leq 2.5|x|^{2}-1.5|x|^{4}, \\
& x^{T} f(x, 2, t)+\frac{7-1}{2}|g(x, 2, t)|^{2}=4|x|^{2}-2|x|^{4},
\end{aligned}
$$

which implies that (4.5) is satisfied with $p=7, \theta=4, \rho=4, A_{1}=2.5, B_{1}=1.5, A_{2}=$ $4, B_{2}=2$. Thus Assumption 7 holds. By a direct computation we know the stationary distribution $\left(\pi_{1}, \pi_{2}\right)=(2 / 3,1 / 3)$ and $\pi A=3$. By virtue of Theorem 4.2 the controlled system (1.3) with any initial value condition

$$
x(t)=x_{0} \in \mathbb{R}, \quad r(t)=i_{0} \in \mathbb{S}, \quad-\tau_{0} \leq t \leq 0,
$$

has a unique global solution $x(t)$ on $[0, \infty)$ which satisfies $\sup _{0 \leq t<\infty} \mathbb{E}|x(t)|^{7}<\infty$.
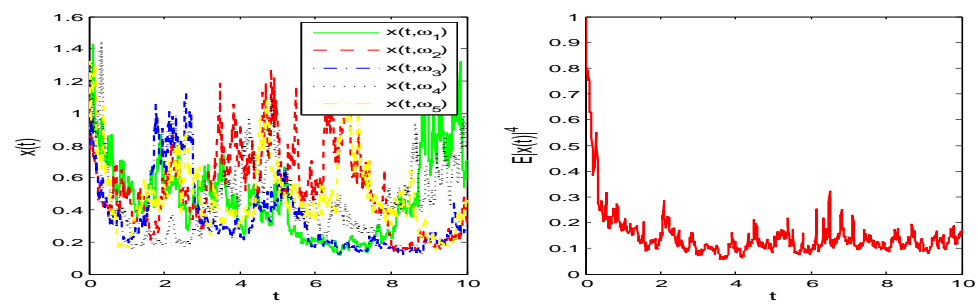

Figure 1: Five sample pathes of the solution $x(t)$ of $(1.1)$ and the sample mean of $|x(t)|^{4}$ for 100 sample points on $t \in[0,10]$ with the initial value $(x(0), r(0))=(1,2)$ and step size $\triangle=10^{-6}$.

In order to have a feeling on the asymptotic behavior we carry out some numerical simulations using MATLAB with the time step size $\triangle=10^{-6}$. Figure 1 depicts 5 sample pathes of the solution and the sample mean of $|x(t)|^{4}$ for 100 sample points, with the initial value $(x(0), r(0))=(1,2)$ for $t \in[0,10]$. One observes from Figure 1 that the solutions is uniformly bounded in the 4 th moment, but the trivial solution $x(t) \equiv 0$ is unstable either $\mathbb{P}$-a.s. or in the moment. So it is necessary to input the feedback control to stabilize SDS (1.1). We will discuss two cases on the design of control functions. In both cases, we will give the range for $\tau+\tau_{0}$ to take and estimate the corresponding Lyapunov exponents.

Case 1 In this case we consider that the state of the underlying SDS and the Markov chain are observable and the feedback control can be input in both modes 1 and 2. Let 
$\alpha(1)=6, \alpha(2)=6$. Then $\pi \alpha=6>\pi A$ and $\kappa_{\alpha-A}=\infty$. By (2.5) and (2.13) we can obtain that $\tau^{\prime}=9.6 \times 10^{-3}$ and $\zeta_{2, \alpha-A}^{\tau^{\prime}}=3.265$. Fix $\sigma=2<3=\zeta_{2, \alpha-A}^{\tau^{\prime}} \wedge(2 \hat{B})$, we may get $\tau_{1}^{* *}(\sigma)=2.78 \times 10^{-4}$, choose $\tau=1 \times 10^{-4}, \tau_{0}=1.7 \times 10^{-4}$, then $\zeta_{2, \alpha-A}^{\tau}=5.8345$. By virtue of Theorem 4.4, the solution of DCSDS (1.3) with the initial condition (5.2) has the properties that

$$
\begin{aligned}
& \limsup _{t \rightarrow \infty} \frac{1}{t} \log \mathbb{E}|x(t)|^{2} \leq-3.8345, \limsup _{t \rightarrow \infty} \frac{1}{t} \log \mathbb{E}|x(t)|^{4} \leq-3.8345 \\
& \int_{0}^{\infty} \mathbb{E}|x(t)|^{6} d t<\infty, \quad \limsup _{t \rightarrow \infty} \frac{1}{t} \log (|x(t)|) \leq-1.9172 \mathbb{P}-\text { a.s. }
\end{aligned}
$$

Figure 2 depicts five sample pathes of the solution $x(t)$ and the sample mean of $|x(t)|^{4}$ for 100 sample points for the controlled system (1.3) for $t \in[0,4]$ with the initial value $(x(0), r(0))=(1,2)$ and step size $\triangle=10^{-6}$.
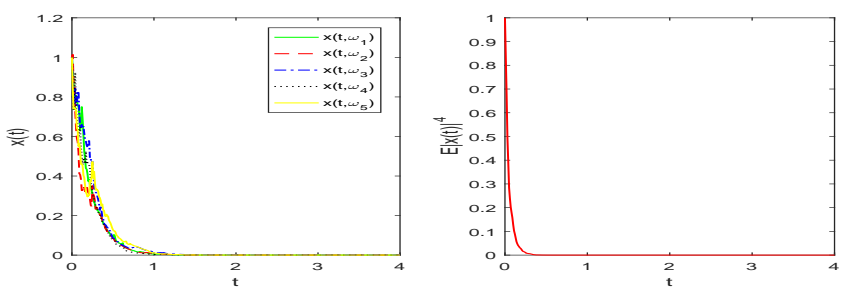

Figure 2: Five sample pathes of the solution $x(t)$ and the sample mean of $|x(t)|^{4}$ for 100 sample points, for the controlled system (1.3) for $t \in[0,4]$ with the initial value $(x(0), r(0))=(1,2)$ and step size $\triangle=10^{-6}$.

Case 2 In this case we consider that the feedback control can only be input to one mode but not the other. Assume that the system in mode 1 is controllable but not in mode 2. Mathematically, we let $\alpha(1)=9, \alpha(2)=0$. Then $\pi \alpha=6$ and $\kappa_{\alpha-A}=3.46$. By (2.5) and (2.13) we can obtain that $\tau^{\prime}=3.73 \times 10^{-3}$ and $\zeta_{2, \alpha-A}^{\tau^{\prime}}=0.5626$. Fix $\sigma=0.5<0.5626=\zeta_{2, \alpha-A}^{\tau^{\prime}} \wedge(2 \hat{B})$, we may get $\tau_{2}^{* *}(\sigma)=5.83 \times 10^{-6}$, choose $\tau=$ $3 \times 10^{-6}, \tau_{0}=2.8 \times 10^{-6}$, then $\zeta_{2, \alpha-A}^{\tau}=1.0747$. By Theorem 4.4, we can then conclude that

$$
\begin{aligned}
& \limsup _{t \rightarrow \infty} \frac{1}{t} \log \mathbb{E}|x(t)|^{2} \leq-0.5747, \limsup _{t \rightarrow \infty} \frac{1}{t} \log \mathbb{E}|x(t)|^{4} \leq-0.5747 \\
& \int_{0}^{\infty} \mathbb{E}|x(t)|^{6} d t<\infty, \quad \limsup _{t \rightarrow \infty} \frac{1}{t} \log (|x(t)|) \leq-0.2874 \mathbb{P}-\text { a.s. }
\end{aligned}
$$

Figure 3 depicts five sample pathes of the solution $x(t)$ and the sample mean of $|x(t)|^{4}$ for 100 sample points for the controlled system (1.3) for $t \in[0,4]$ with the initial value $(x(0), r(0))=(1,2)$, step size $\triangle=10^{-8}$. Due to the definition of $\beta_{3}(\cdot)$ in (4.23) one observes that the balanced control values $\alpha(\cdot)$ in modes are helpful to get a better lower bound of $\tau^{*}$ or $\tau^{* *}$, and this is illustrated in this example.

\section{References}

[1] W.J. Anderson, Continuous Markov chains, Springer, New York, 1991. 

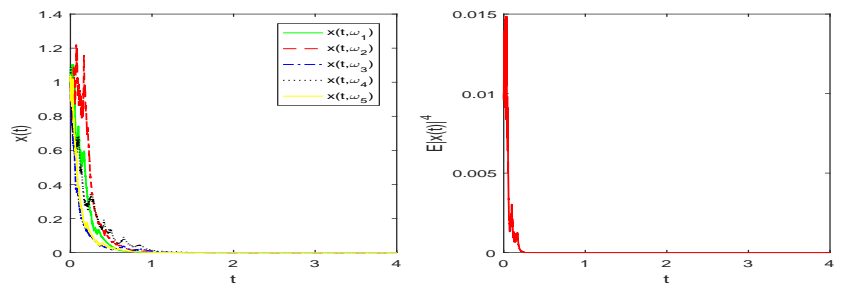

Figure 3: Five sample pathes of the solution $x(t)$ and the sample mean of $|x(t)|^{4}$ for 100 sample points for the controlled system (1.3) where the control is only input to the system in mode 1 , for $t \in[0,4]$ with the initial value $(x(0), r(0))=(1,2)$ and step size $\triangle=10^{-8}$.

[2] A. Arapostathis, V.S. Borkar and M.K. Ghosh, Ergodic control of diffusion processes, Cambridge University Press, Cambridge, 2012.

[3] J.B. Bardet, H. Guérin, F. Malrieu, Long time behavior of diffusions with Markov switching, ALEA Lat. Am. J. Probab. Math. Stat., 7 (2010), pp. 151-170.

[4] A.B. Chammas and C.T. Leondes, On the finite time control of linear systems by piecewise constant output feedback, Internat. J. Control, 30 (1979), pp. 227-234.

[5] T. Hagiwara and M. Araki, Design of stable state feedback controller based on the multirate sampling of the plant output, IEEE Trans. Automat. Control, 33 (1988), pp. 812-819.

[6] T. Hagiwara and M. Araki, On preservation of strong stabilizability under sampling. IEEE Trans. Automat. Control, 33 (1988), pp. 1080-1082.

[7] J.C. Geromel and G.W. Gabriel, Optimal H2 state feedback sampled-data control design of Markov jump linear systems, Automatica, 54 (2015), pp. 182-188.

[8] X. Li, X. Mao, Stabilisation of highly nonlinear hybrid stochastic differential delay equations by delay feedback control, Automatica, 112 (2020), 108657.

[9] Y. Li, J. Lu, X. Mao and Q. Qiu, Stabilization of hybrid systems by feedback control based on discrete-time state and mode observations, Asian J. Control 19(6) (2017), pp.1943-1953.

[10] Z. Lu, J. Hu, and X. Mao, Stabilisation by delay feedback control for highly nonlinear hybrid stochastic differential equations. Discrete and Continuous Dynamical Systems - Series B., 24 (2019), pp. 4099-4116.

[11] X. Mao, Stochastic Differential Equations and Applications, 2nd Edition, Chichester: Horwood, 2008.

[12] X. Mao, Stabilization of continuous-time hybrid stochastic differential equations by discretetime feedback control, Automatica, 49 (2013), pp. 3677-3681.

[13] X. Mao, L. James, L. Huang, Stabilisation of hybrid stochastic differential equations by delay feedback control, System and Control Letter, 57 (2008), pp. 927-935. 
[14] X. Mao, W. Liu, L. Hu, Q. Luo, and J. Lu, Stabilization of hybrid stochastic differential equations by feedback control based on discrete-time state observations, Systems Control Lett., 73 (2014), pp. 88-95.

[15] X. Mao, C. Yuan, Stochastic Differential Equations with Markovian Switching, Imperial College Press, 2006.

[16] Q. Qiu, W. Liu, L. Hu, X. Mao, S. You, Stabilization of stochastic differential eqautions with Markovian switching by feedback control based on discrete-time state observation with a time delay, Statistics and Probability Letters, 112 (2016), pp. $16-26$.

[17] M. Pinsky, R.G. Pinsky, Transience/recurrence and central limit theorem behavior for diffusions in random temporal environments, Ann. Probab. 21 (1993), pp. 433452.

[18] M. Pinsky, M. Scheutzow, Some remarks and examples concerning the transience and recurrence of random diffusions, Ann. Inst. H. Poincaré Probab. Statist. 28 (1992), pp. 519-536.

[19] M.L. Rosinberg, T. Munakata, and G. Tarjus, Stochastic thermodynamics of Langevin systems under time-delayed feedback control: Second-law-like inequalities, Phys. Rev. E 91 (2015), 042114.

[20] J. Shao, Strong solutions and strong Feller properties for regime-switching diffusion processes in an infinite state space, SIAM J. Control Optim., 53 (2015), pp. 24622479 .

[21] J. Shao, Stabilization of regime-switching processes by feedback control based on discrete time observations. SIAM J. Control Optim. 55 (2017), pp. 724-740.

[22] J. Shao and F. Xi, Stabilization of regime-switching processes by feedback control based on discrete time observations II: State-dependent case. SIAM J. Control Optim. 57 (2019), pp. 1413-1439.

[23] A.N. Shiryaev, Probability 2nd edition, World Book Inc., 2004.

[24] G. Song, B. Zheng, Q. Luo, X. Mao, Stabilisation of hybrid stochastic differential equations by feedback control based on discrete-time observations of state and mode, IET Control Theory Appl., 11 (2017), pp. 301-307.

[25] G. Yin and F. Xi, Stability of regime-switching jump diffusions, SIAM J. Control Optim., 48 (2010), pp. 4525-4549.

[26] G. Yin and C. Zhu,Hybrid Switching Diffusions. Properties and Applications, Springer, New York, 2010.

[27] S. You, W. Liu, J. Lu, X. Mao, and Q. Qiu, Stabilization of hybrid systems by feedback control based on discrete-time state observations, SIAM J. Control Optim., 53 (2015), pp. 905-925. 